\title{
Effect of Peak Inert-Mode Temperature on Elemental Carbon Measured Using Thermal-Optical Analysis
}

\author{
Ramachandran Subramanian, ${ }^{1, \dagger}$ Andrey Y. Khlystov, ${ }^{3}$ and Allen L. Robinson ${ }^{1,2}$ \\ ${ }^{I}$ Department of Mechanical Engineering, Carnegie Mellon University, Pittsburgh, Pennsylvania, USA \\ ${ }^{2}$ Department of Engineering and Public Policy, Carnegie Mellon University, Pittsburgh, \\ Pennsylvania, USA \\ ${ }^{3}$ Department of Civil and Environmental Engineering, Duke University, Durham, North Carolina, USA
}

\begin{abstract}
Thermal-optical analysis is a conventional method for classifying carbonaceous aerosols as organic carbon $(O C)$ and elemental carbon (EC). This article examines the effects of three different temperature protocols on the measured EC. For analyses of parallel punches from the same ambient sample, the protocol with the highest peak helium-mode temperature $\left(8^{\circ}{ }^{\circ} \mathrm{C}\right)$ gives the smallest amount of EC, while the protocol with the lowest peak helium-mode temperature $\left(550^{\circ} \mathrm{C}\right)$ gives the largest amount of EC. These differences are observed when either sample transmission or reflectance is used to define the OC/EC split. An important issue is the effect of the peak helium-mode temperature on the relative rate at which different types of carbon with different optical properties evolve from the filter. Analyses of solvent-extracted samples are used to demonstrate that high temperatures $\left(870^{\circ} \mathrm{C}\right)$ lead to premature $\mathrm{EC}$ evolution in the helium-mode. For samples collected in Pittsburgh, this causes the measured EC to be biased low because the attenuation coefficient of pyrolyzed carbon is consistently higher than that of EC. While this problem can be avoided by lowering the peak helium-mode temperature, analyses of wood smoke dominated am-
\end{abstract}

Received 30 June 2005; accepted 28 February 2006.

The authors would like to acknowledge the many useful conversations with B.J. Turpin at Rutgers University. Dr. Eric Lipsky provided the tunnel samples. Erin Casgren-Tindall, Prakash Rao, Leonard Lucas, David Wynne, Michael Quirolo, Mark Prack, Jessica Chiu, and Raymond Obico analyzed the vast majority of the filters for the intercomparison studies. RS thanks Tami Bond for time off to finish this paper. This research was conducted as part of the Pittsburgh Air Quality Study, which was supported by US Environmental Protection Agency under contract R82806101 and the US Department of Energy National Energy Technology Laboratory under contract DE-FC26-01NT41017. This work was supported in part by the Pennsylvania Infrastructure Technology Alliance, a partnership of Carnegie Mellon, Lehigh University, and the Commonwealth of Pennsylvania's Department of Community and Economic Development (DCED). This paper has not been subject to EPA's required peer and policy review, and therefore does not necessarily reflect the views of the Agency. No official endorsement should be inferred.

Address correspondence to Allen L. Robinson, Department of Mechanical Engineering, 5000 Forbes Ave., Carnegie Mellon University, Pittsburgh, PA 15213, USA. E-mail: alr@andrew.cmu.edu

${ }^{\dagger}$ Now at the Department of Civil and Environmental Engineering, University of Illinois, Urbana, IL 61801, USA. bient samples and levoglucosan-spiked filters indicate that too low helium-mode peak temperatures $\left(5^{\circ} 0^{\circ} \mathrm{C}\right)$ allow non-light absorbing carbon to slip into the oxidizing mode of the analysis. If this carbon evolves after the OC/EC split, it biases the EC measurements high. Given the complexity of ambient aerosols, there is unlikely to be a single peak helium-mode temperature at which both of these biases can be avoided.

\section{INTRODUCTION}

The carbonaceous component of fine particulate matter is commonly classified as organic carbon (OC) and elemental carbon (EC). A variety of thermal or thermal-optical measurement techniques have been developed to measure particulate $\mathrm{OC}$ and EC (Malissa, Puxbaum, and Pell 1976; Huntzicker et al. 1982; Chow et al. 1993; Kerminen et al. 1997). While these methods generally measure the same amount of total carbon, the OC/EC split is operationally defined with significant differences in the amount of OC and EC measured by different methods (Chow et al. 2001; Schmid et al. 2001; Fung, Chow, and Watson 2002).

This article largely focuses on the thermal-optical transmission (TOT) technique first developed by Huntzicker et al. (1982), with subsequent developments reported by Birch and Cary (1996). This technique is widely used in the atmospheric science community, including the EPA's Speciation Trends Network (STN). Briefly, a filter sample is exposed to a prescribed temperature protocol first in an inert (helium, He) environment and then in an oxidizing (helium and oxygen mixture, $\mathrm{He} / \mathrm{Ox}$ ) atmosphere. Ideally, OC volatilizes in the He-mode of the analysis, while EC combusts in the oxidizing atmosphere at high temperatures. A major complication is that a fraction of the OC chars or pyrolyzes in the inert mode of the analysis. The residual carbon remaining on the filter after heating in the inert mode is called pyrolyzed organic carbon (PC), and can absorb light and requires an oxidizing atmosphere to evolve off the filter, similar to EC. Not all thermally altered OC absorbs light; however, in this article the term PC is used to refer only to light-absorbing pyrolyzed carbon. The term "non-light absorbing carbon" is used to refer to unaltered OC and thermally altered OC that does not 
absorb light. Not accounting for PC formation can significantly bias the OC/EC split. To account for pyrolysis, the transmission of a laser light through the sample is monitored and used to split the total carbon into OC and EC. A variant on this approach is the thermal-optical reflectance (TOR) method which uses reflected laser light to determine the OC/EC split.

Previous studies have demonstrated that changing the temperature protocol can alter the EC measured by thermal-optical analysis (Chow et al. 2001; Yang and Yu 2002; Conny et al. 2003; Schauer et al. 2003; Chow et al. 2004). Lowering the temperatures of the steps in the He-mode can increase the measured EC; this increase has been attributed to OC not completely evolving or not pyrolyzing into light-absorbing carbon (Conny et al. 2003; Schauer et al. 2003). While previous research has demonstrated that certain types of OC such as wood smoke do not completely evolve or pyrolyze at low inert-mode temperatures (Novakov and Corrigan 1995), the effect of this on the thermaloptical EC is not well understood. Too high of a peak inert-mode temperature is also thought to bias the OC/EC split due to premature EC evolution (Chow et al. 2001). At high temperatures mineral oxides can promote combustion of EC in an inert environment (Fung 1990); however, it has not been demonstrated that the light-absorbing carbon evolving at high temperatures in the He-mode is EC, and why this should alter the measured $\mathrm{EC}$ is unclear. An additional challenge is that the effects of temperature protocol on the measured EC can vary from sample to sample, presumably due to differences in sample composition (Schauer et al. 2003).

While a number of hypotheses have been put forward to explain the observed changes in thermal-optical EC measured with different temperature protocols, the underlying causes of the changes are not well understood. Yang and Yu (2002) indicate that changes in measured EC with temperature protocol are related to the attenuation coefficients of PC and EC. Chow et al. (2004) proposes that the effects of changing temperature protocol on the measured EC may be mitigated by using TOR as opposed to TOT to define the OC/EC split. A critical factor in explaining the variability in the measurements is the highest temperature step in the He-mode (Conny et al. 2003).

This article examines the effect of the peak He-mode temperature (step 4 of the Helium mode, He4) on the OC/EC split defined by thermal-optical analysis. Both ambient and sourcedominated samples were analyzed using three different temperature protocols: two versions of the NIOSH 5040 protocol (NIOSH 1999) and a version of the IMPROVE protocol (Chow et al. 1993). The NIOSH protocols have peak He-mode temperatures of 870 and $700^{\circ} \mathrm{C}$ while the IMPROVE-type protocol has a peak He-mode temperature of $550^{\circ} \mathrm{C}$. A combination of solvent extraction to minimize pyrolysis, analysis of filters doped with model OC mixtures, and quantitative analysis of optical data is used to evaluate the fundamental assumptions behind thermal-optical OC/EC analysis and to investigate the causes of differences in the EC measured using different temperature protocols. While most of the research uses sample transmission
(TOT) to define the OC/EC split, we also report results from a number of analyses based on sample reflectance (TOR).

\section{METHODS}

\section{Sample Collection and Analysis}

Ambient $\mathrm{PM}_{2.5}$ samples were collected at an urban park near the Carnegie Mellon University campus in Pittsburgh, PA as part of the Pittsburgh Air Quality Study (PAQS) (Wittig et al. 2004). Mobile source dominated samples were collected using the same samplers in the ventilation duct of the Squirrel Hill tunnel on Interstate-376 in Pittsburgh (Grieshop et al. 2006). Details of the design and operation of the two organic samplers are provided in Subramanian et al. (2004). Briefly, one sampler used a quartz fiber filter (Gelman Tissuquartz 2500 QAO-UP) downstream of a sharp cut $\mathrm{PM}_{2.5}$ cyclone. The second sampler had a quartz filter downstream of an activated carbon monolith denuder (MastCarbon Ltd, UK) and a sharp-cut $\mathrm{PM}_{2.5}$ cyclone. The majority of the samples were collected for 24 hours, with a limited number of shorter and longer duration samples. All the quartz filters were baked at $550^{\circ} \mathrm{C}$ in air for at least four hours prior to use. The data presented here are not blank-corrected since we compare paired punches from the same samples, and blank correction does not affect the results significantly.

The samples were analyzed using a Sunset Laboratory Thermal/Optical Carbonaceous Aerosol Analyzer. The carbon analyzer was routinely checked for leaks and operated using Ultra-High Purity (Grade 6.0) Helium gas with two oxygen traps installed on the inlet line to avoid problems with oxygen contamination. The instrument is calibrated prior to use with a combination of sucrose standards and manual methane injections. The OC/EC split is defined by the sample transmission measured at $680 \mathrm{~nm}$. Any carbon evolving after the laser signal reaches $97 \%$ of the initial, pre-pyrolysis value is classified as EC. A value of 97\% was used to be consistent with the default value in the Sunset Laboratories data analysis program. The calculation procedures for defining the OC/EC split used the algorithm as described by Sunset Labs (David Smith, personal communication, 2002).

Table 1 lists the three temperature profiles. Two are based on the NIOSH 5040 protocol (NIOSH 1999); they are identical except that one has a peak He-mode temperature (He4) of $870^{\circ} \mathrm{C}$ and the second a peak He-mode temperature of $700^{\circ} \mathrm{C}$ (typical thermograms from these two protocols are shown in Figure 1). The third protocol is an implementation of the IMPROVE protocol, with a peak He-mode temperature of $550^{\circ} \mathrm{C}$ (Chow et al. 1993). We refer to these protocols as the He4-870, He4700 , and $\mathrm{He} 4-550.700^{\circ} \mathrm{C}$ is an intermediate temperature between the NIOSH and IMPROVE protocols. This value was chosen to be more consistent with the original Huntzicker protocol (Huntzicker et al. 1982; Turpin 1989); it is higher $\left(700^{\circ} \mathrm{C}\right.$ versus $650^{\circ} \mathrm{C}$ ) to account for the differences in thermocouple locations in the two analyzers (B.J. Turpin, personal communication). These protocols are based on the temperature measured by a thermocouple located immediately downstream of the sample 
TABLE 1

Temperature protocols used to analyze the samples in this article

\begin{tabular}{|c|c|c|c|c|c|}
\hline \multirow[b]{2}{*}{ Step } & \multicolumn{3}{|c|}{ Based on NIOSH $5040^{a}$} & \multicolumn{2}{|c|}{$\mathrm{He} 4-550^{b}$} \\
\hline & Duration (s) & $\mathrm{He} 4-870 \mathrm{~T}\left({ }^{\circ} \mathrm{C}\right)$ & $\mathrm{He} 4-700 \mathrm{~T}\left({ }^{\circ} \mathrm{C}\right)$ & Duration (s) & $\mathrm{T}\left({ }^{\circ} \mathrm{C}\right)$ \\
\hline He1 & 120 & 340 & 340 & 120 & 120 \\
\hline $\mathrm{He} 2$ & 120 & 500 & 500 & 120 & 250 \\
\hline He3 & 120 & 615 & 615 & 120 & 450 \\
\hline $\mathrm{He} 4$ & 180 & 870 & 700 & 120 & 550 \\
\hline (Helium) & 45 & (cool down) & (cool down) & N/A & N/A \\
\hline $\mathrm{HeOx} 1$ & 45 & 575 & 575 & 300 & 550 \\
\hline $\mathrm{HeO} 2$ & 45 & 650 & 650 & 240 & 700 \\
\hline $\mathrm{HeO} \times 3$ & 45 & 725 & 725 & 240 & 800 \\
\hline $\mathrm{HeOx} 4$ & 45 & 800 & 800 & 180 & 910 \\
\hline $\mathrm{HeO} 55$ & 100 & 910 & 910 & N/A & N/A \\
\hline Internal standard & 110 & - & - & 110 & - \\
\hline
\end{tabular}

${ }^{a}$ NIOSH (1999).

${ }^{b}$ Based on IMPROVE protocol (Chow et al. 1993). N/A = not applicable. The IMPROVE protocol does not have a cool-down step or a $\mathrm{HeO} 5$ step.

(the standard design of the Sunset OC/EC analyzer), which may not be exactly equal to the sample temperature. For a limited number of samples, we also lengthened the He/Ox temperature steps (without changing the temperatures listed in Table 1) to investigate the influence of duration of the different temperature steps on the OC/EC split.

We adapted the solvent treatment method of Fung (1990) to extract a portion of the organic carbon before analysis to minimize pyrolysis and thus reduce the PC interference in the optically-defined OC/EC split. Selected filter punches were immersed in a methylene chloride: acetone: hexane mixture (2:4:4 by volume) for an hour (the solvent mixture is replaced after 30 minutes), with periodic gentle stirring. The punches are then rinsed with methylene chloride and dried in an oven at $50^{\circ} \mathrm{C}$ for 30 minutes before OC/EC analysis. The average OC on four pre-baked blanks solvent-treated in this manner was $0.08 \mu \mathrm{g}$ $\mathrm{C} / \mathrm{cm}^{2}$; all four values were below the instrument detection limit for $\mathrm{OC}\left(0.2 \mu \mathrm{g}-\mathrm{C} / \mathrm{cm}^{2}\right)$.

\section{Calculation of the Attenuation Coefficient of Evolved Carbon}

The changes in the laser transmission during OC/EC analysis depend on the mass and the attenuation coefficient of the carbon evolving from the filter. The attenuation coefficient $(\mathrm{k})$ of the carbon that evolves from different segments of the analysis (e.g., the net attenuation coefficient of the $\mathrm{He} / \mathrm{Ox}$ carbon) is calculated using the Lambert-Beer law:

$$
k=\frac{-\ln (I o / I)}{C}
$$

where $I o$ and $I$ are the initial and final laser transmission through the sample and filter for the portion of the analysis of interest and $C$ is the mass of carbon per unit filter area that is lost from (or combusts off) the filter during the analysis segment; thus, Io is the laser transmission through the loaded filter, not the blank. Note that this is the reverse of the more-conventional Aethalometer approach (e.g., Gundel et al. 1984).

When analyzing the optical data, one must be careful to account for potential effects of filter loading. This dependence is illustrated in Figure 2, which shows the net laser attenuation of the He/Ox carbon for samples collected in a highway tunnel as a function of filter loading. For low filter loadings (less than $15 \mu \mathrm{g}-\mathrm{C} / \mathrm{cm}^{2}$ ), the net laser attenuation increases more or less linearly with loading and Equation (1) yields consistent values for the net specific attenuation coefficient of the He/Ox carbon $\left(\sim 20 \mathrm{~m}^{2} / \mathrm{g}-\mathrm{C}\right)$. However, at higher filter loadings, the carbon on heavily loaded filters appears lighter than the carbon on more lightly loaded filters. This effect is referred to as a "shadowing" or "light-pipe" effect (Gundel et al. 1984; Weingartner et al. 2003). The He/Ox carbon loadings of the filters considered in this article are less than $15 \mu \mathrm{g}-\mathrm{C} / \mathrm{cm}^{2}$ to minimize the "shadowing" or "light-pipe" effect.

\section{Instrument Precision}

The precision of the OC/EC analyzer was evaluated by analyzing multiple punches from three 102-mm filter $\mathrm{PM}_{2.5}$ samples collected with a medium-volume sampler and parallel punches from four 47-mm samples using the same protocol. The absolute EC (EC in $\left.\mu \mathrm{g}-\mathrm{C} / \mathrm{cm}^{2}\right)$ measured with the same protocol on our Sunset instrument showed a precision within $7 \%$ (the maximum relative standard deviation (RSD) observed within each of the three analysis protocols with untreated ambient samples); similarly, the TC precision is $3 \%$ across all protocols. The maximum 

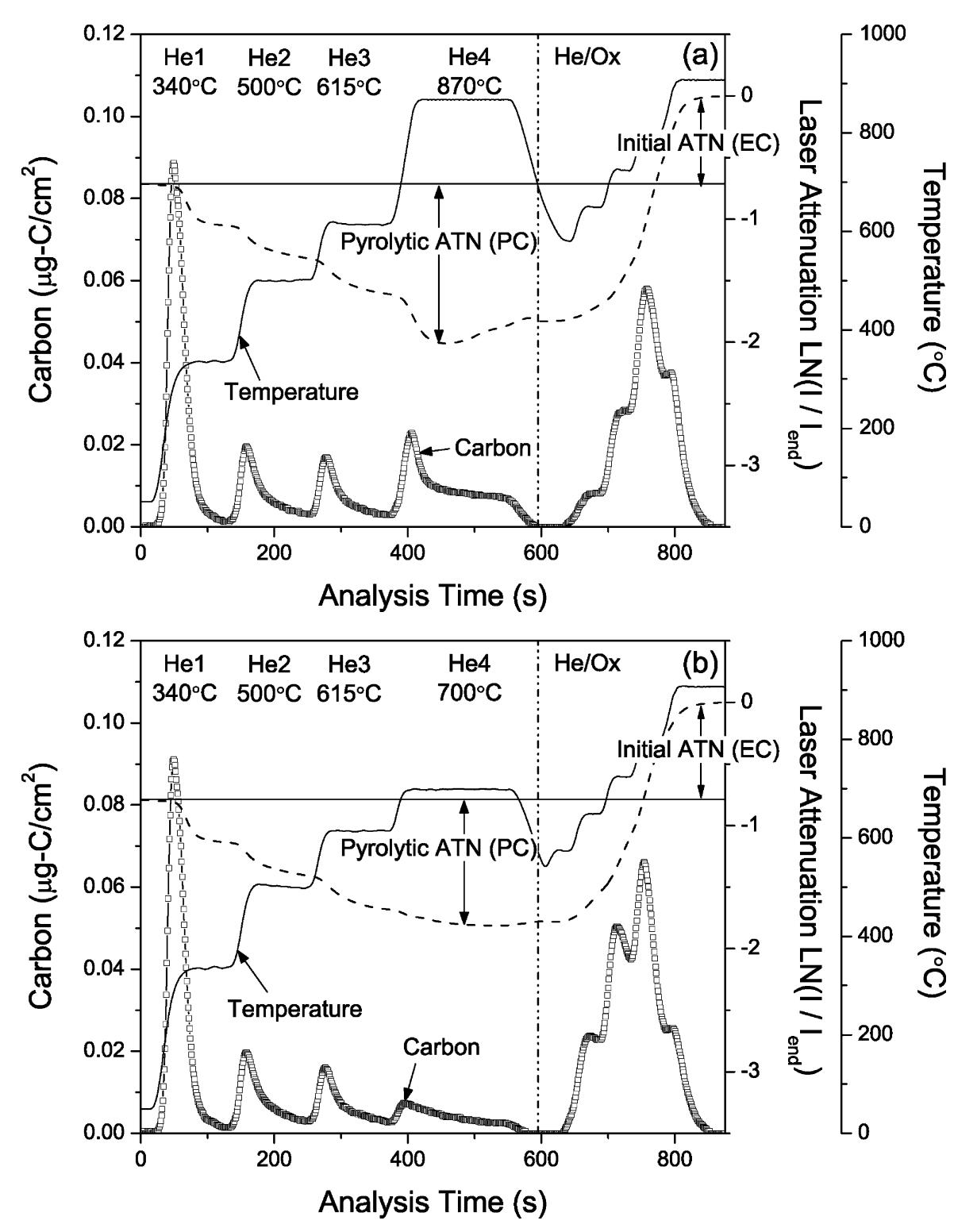

FIG. 1. Thermograms from the analysis of a typical ambient sample with the (a) He4-870 and (b) He4-700 protocols. The thermograms are for parallel punches of the same filter. Dashed line indicates laser attenuation. There is clear loss of light-absorbing carbon in the He-mode of the He4-870 protocol but not in the He-mode of the He4-700 protocol.

RSD in the EC/TC ratios within each protocol was $6 \%$. This performance is consistent with the Sunset Laboratory reported measurement precision of $\pm 5 \%$ for the same instrument, with a minimum uncertainty of $0.2 \mu \mathrm{g}-\mathrm{C} / \mathrm{cm}^{2}$ (Cary 2003). Schauer et al. (2003) report the precision as $4-13 \%$ for OC and between 6-21\% for EC between different instruments running the same temperature-time protocol on parallel punches.

\section{RESULTS}

We first consider results from samples analyzed using the He4-870 protocol. As part of the Supersite study, EPA requested that the He4-870 protocol be used as part of the carbon analysis; therefore, essentially all of the PAQS samples were analyzed with this protocol. We then compare the results from analyses using the He4-870 and He4-700 protocols and critically evaluate the fundamental assumptions underlying TOT analysis in order to explain the differences between the measurements. Finally, we consider the effects of even lower peak He-mode temperatures by comparing results from the He4-700 and He4-550 protocols. Only limited subsets of the PAQS samples were analyzed with the He4-550 and He4-700 protocols for comparison purposes.

A typical thermogram from the analysis of a filter punch using the He4-870 protocol is shown in Figure 1(a). The formation of light-absorbing pyrolyzed carbon (PC) causes the transmission of laser light through the sample (and filter) to decrease 


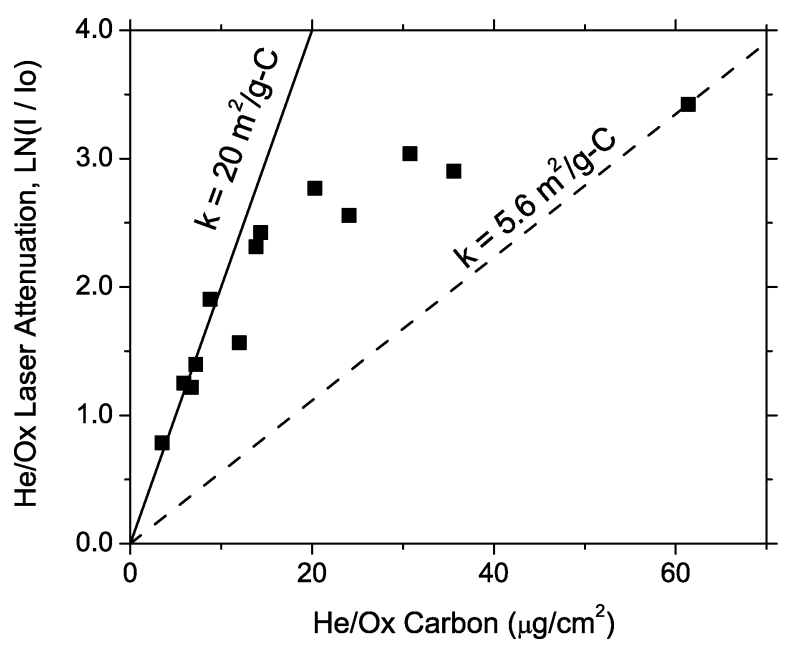

FIG. 2. Net change in laser attenuation over the He/Ox mode of samples collected in a highway tunnel, as a function of filter loading. Lightly loaded filters yield a consistent estimate of the specific attenuation coefficient, $20 \mathrm{~m}^{2} / \mathrm{g}-\mathrm{C}$, indicated by the solid line. At high filter loadings the specific attenuation coefficient appears to decrease, as indicated by the dashed line, due to the "shadowing" or "light-pipe" effect.

through much of the He-mode of the analysis. The transmission reaches a minimum value partway through the $870^{\circ} \mathrm{C}$ temperature step of the He-mode and then begins increasing, indicating premature evolution of light-absorbing carbon. Over 66\% of the almost 500 PAQS samples (both denuded and undenuded) analyzed with the He4-870 protocol exhibited similar loss of light-absorbing carbon in the He4 step. Although there is loss of light-absorbing carbon in the He-mode, the OC/EC split occurred in the $\mathrm{He} / \mathrm{Ox}$ mode for more than $99 \%$ of these samples. The loss of light-absorbing carbon in the inert mode of the analysis is not unique to Pittsburgh samples (e.g., Chow et al. 2001; Peterson and Richards 2002).

In the event of loss of light-absorbing carbon in the He-mode of the analysis, a standard recommendation is to reduce the peak temperature of this mode (NIOSH 1999). Recently, Conny et al. (2003) proposed shortening the duration of the He4 step to avoid the loss of light-absorbing carbon in the He-mode. This is an interesting approach that links the temperature and duration of the $\mathrm{He} 4$ step, essentially by switching to the $\mathrm{He} / \mathrm{Ox}$ mode when the laser reaches its minimum transmission. A complication is that this time will vary with sample loading, sample composition, and He4 temperature, which makes it difficult to use this approach given the large number of samples considered in this study.

Figure 1(b) shows a thermogram from the He4-700 analysis of a second punch from the same filter shown in Figure 1(a). The laser signal reaches its minimum value in the He4 step $\left(700^{\circ} \mathrm{C}\right)$ and does not increase until the He/Ox-mode suggesting no loss of light-absorbing carbon in the He-mode. However, a flat laser signal in the $\mathrm{He} 4$ step does not guarantee that light-absorbing carbon is not lost in the He-mode because the loss of light-absorbing carbon could be offset by continued pyrolysis.

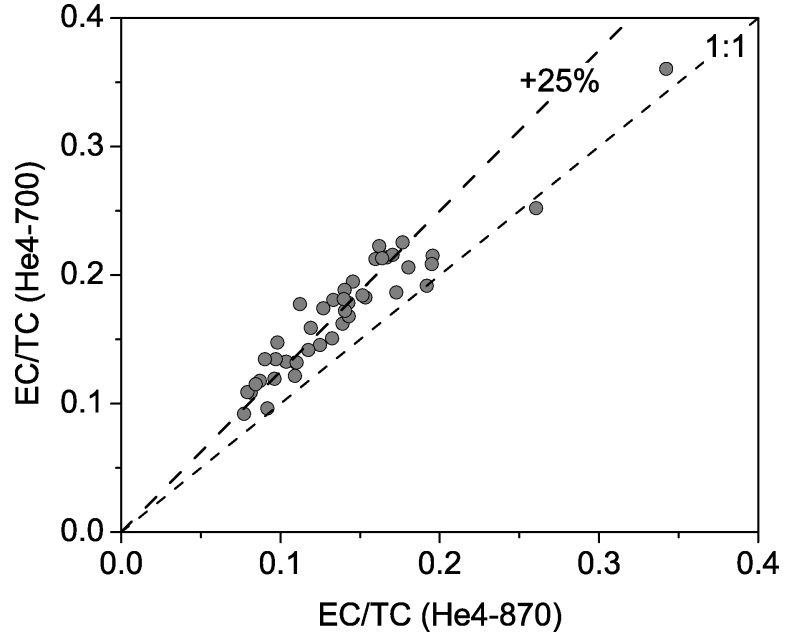

FIG. 3. Scatter plot of the EC (as a fraction of TC) measured by the He4-870 and He4-700 temperature protocols for parallel punches taken from 43 different ambient samples including 24-hour denuded samples and undenuded samples with sampling durations of $8,24,48$, or 96 hours. The He4-700 protocol gives $25 \pm 13 \%$ (average \pm standard deviation) higher EC/TC ratios than the He4870 protocol. The maximum EC loading for these samples is $8.4 \mu \mathrm{g}-\mathrm{C} / \mathrm{cm}^{2}$ (measured by the He4-700 protocol).

Figure 3 compares the TOT-EC measured on parallel punches of 43 different filters analyzed using the He4-700 and He4-870 protocols, the comparison set including both denuded and undenuded samples as well as different sampling durations $(8,24$, 48 , and 96 hours). Comparisons are made in terms of the EC/TC ratio to account for any minor variations in total carbon loadings of the parallel filter punches. During the analysis of all of these samples the laser transmission increased in the He4 step of the He4-870 protocol, indicating premature loss of light absorbing carbon. This rise in the sample transmission during the He4 step is comparatively much smaller with the He4-700 protocol, as the laser reaches a minimum value in the He4 step and remains close to this value till the end of the He-mode for most samples.

The EC/TC ratio of the punches analyzed with the He4-700 protocol is $25 \pm 13 \%$ (average \pm standard deviation) higher than the EC/TC ratio measured on parallel punches from the same filter using the He4-870 protocol. Both the average bias and the variability in the bias are significantly larger than the $6 \%$ instrument precision for EC/TC measurements made on parallel punches from the same sample analyzed with the same protocol. This indicates that the bias is statistically significant and that there is some sample-to-sample variability in the bias, likely associated with sample composition. The maximum bias is 58\%, while the EC/TC ratio measured with the He4-870 protocol is greater than that measured with the He4-700 protocol for only one sample (26\% compared to $25 \%$ ).

The difference between TOT-EC measured with the He4700 and He4-870 protocols is not due to inhomogeneous filter loadings. For the 43 ambient samples included in the intercomparison, parallel punches from the same filter analyzed with the 

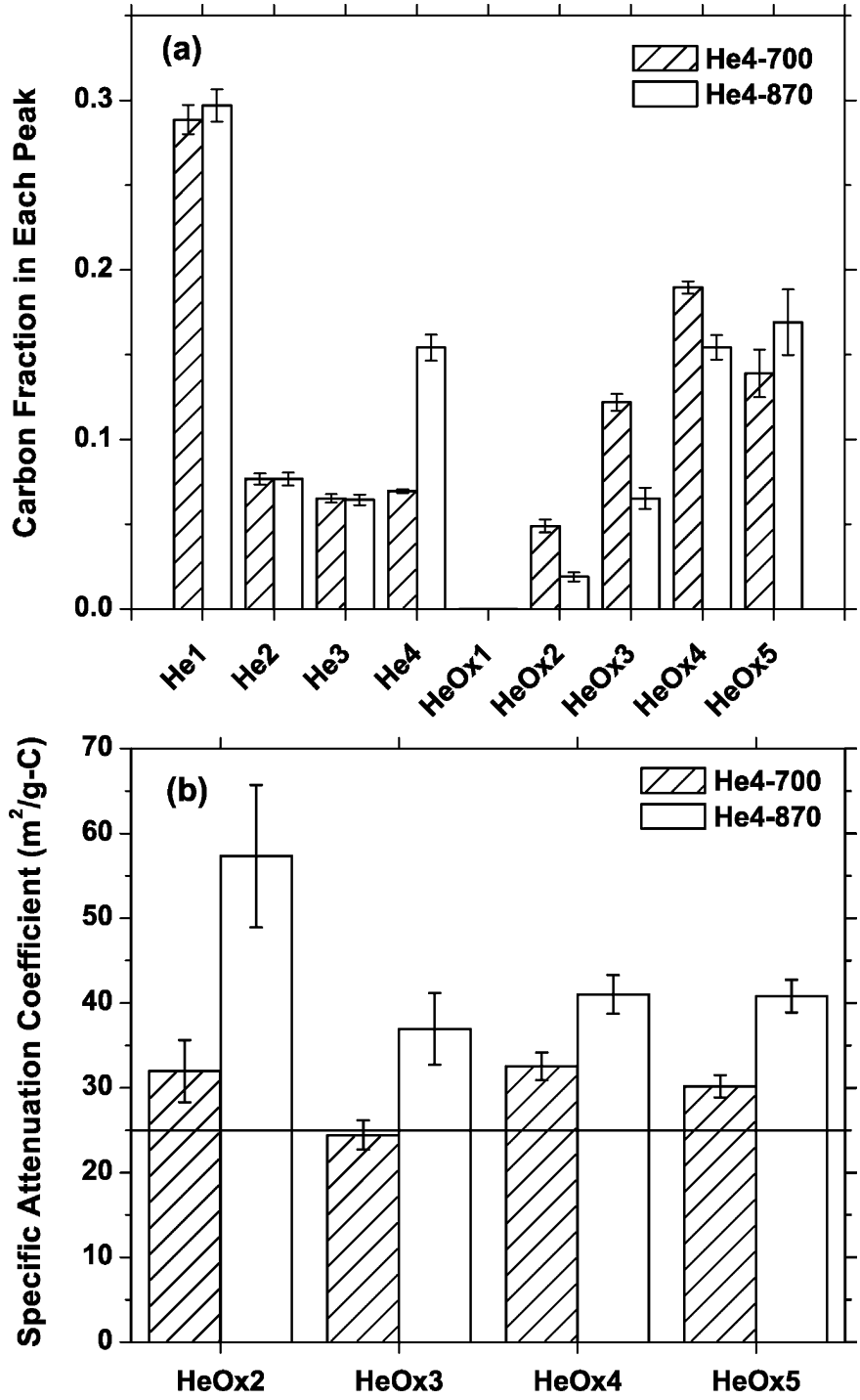

FIG. 4. Average (a) fraction of total carbon that evolves at each temperature step and (b) specific attenuation coefficient of carbon evolving at each temperature step in the He/Ox mode, for twelve undenuded 24-h samples analyzed using both the He4-700 and He4-870 temperature protocols. The horizontal line in (b) indicates an upper bound for $k_{\mathrm{EC}}$, the attenuation coefficient of elemental carbon $\left(25 \mathrm{~m}^{2} / \mathrm{g}-\mathrm{C}\right)$, as discussed in the text. Error bars indicate one standard error of the mean.

H4-870 and He4-700 protocols had TC loadings within 5\% of each other without any systematic differences. A 2-tailed pairedsample t-test of the difference between the final and initial laser transmission indicated that net laser attenuation (i.e., the optical EC loading) was not significantly different between the paired punches analyzed by the two protocols $(p=0.4680)$.

In addition to changing the TOT-EC, there are significant differences in the carbon evolution and laser attenuation of paired punches from the same sample that are analyzed with the He4-870 and He4-700 protocols. Figures 4-6 present averages and scatter plots of carbon evolution pattern and specific attenuation coefficients of samples used in the intercomparison.
Values are shown for different temperature steps of the analysis protocol.

Although there is essentially no difference in the total carbon measured on parallel punches, changing the temperature protocol systematically shifts when carbon evolves from the filter. Figures $4 \mathrm{a}$ and 5 indicate that increasing the peak He-mode temperature shifts carbon from the $\mathrm{He} / \mathrm{Ox}$ to the He-mode. For example, almost twice as much carbon evolves in He4 step of the He4-870 protocol compared to the He4-700 protocol. From the perspective of the He4-700 protocol, more carbon evolves in the lower temperature steps of the He/Ox-mode from the He4700 protocol compared to the He4-870 protocol. The OC/EC split is almost always defined in one of the lower temperature steps of the He/Ox mode, so these shifts in carbon evolution are likely related to the change in the TOT-EC.

Significant differences also exist in the specific attenuation coefficients of the carbon that evolves in the He/Ox mode of samples analyzed with the two protocols. Figure $4 \mathrm{~b}$ shows that, on average, the He/Ox carbon that evolves during the He4870 protocol is significantly darker (i.e., has a higher specific attenuation coefficient) than the carbon coming off the filter in the He/Ox mode of the He4-700 protocol. Figure 6 shows that these trends are consistent across the entire set of samples except for the lower-temperature steps of the heavily loaded multi-day (48-h and 96-h) undenuded samples where we might be seeing the saturation effect illustrated in Figure 2. The changes in sample attenuation may be related to the changes in carbon evolution shown in Figures 4a and 5; for example, if increasing the peak temperature of the He-mode preferentially shifted carbon with a lower attenuation coefficient into the He-mode.

\section{Evaluation of Assumptions Underlying the Optical Correction}

The systematic bias in the TOT-EC measured with the He4870 and He4-700 protocols indicates that we must carefully evaluate the fundamental assumptions underlying the optically defined EC. In order for thermal-optical analysis to correctly define the OC/EC split requires that either (1) PC and non-lightabsorbing carbon evolve completely before EC, or (2) the carbon evolving after the OC/EC split has the same attenuation coefficient as EC (Yang and Yu 2002). If both assumptions are invalid then the TOT method will misclassify the OC/EC split.

To evaluate these assumptions, carbon analyses were performed on filter punches that had been solvent extracted. Figure 7 compares thermograms of extracted and untreated punches taken from the same filter. Figure 7a shows results from a 24-h denuded sample analyzed with the He4-870 protocol, and Figure $7 \mathrm{~b}$ shows results from a $24-\mathrm{h}$ undenuded sample collected on the same day analyzed using the He4-700 protocol.

Figure 7 indicates that solvent extraction removes a large fraction of the carbon from the sample, which, in turn, reduces the formation of PC. Solvent extraction significantly reduces, 


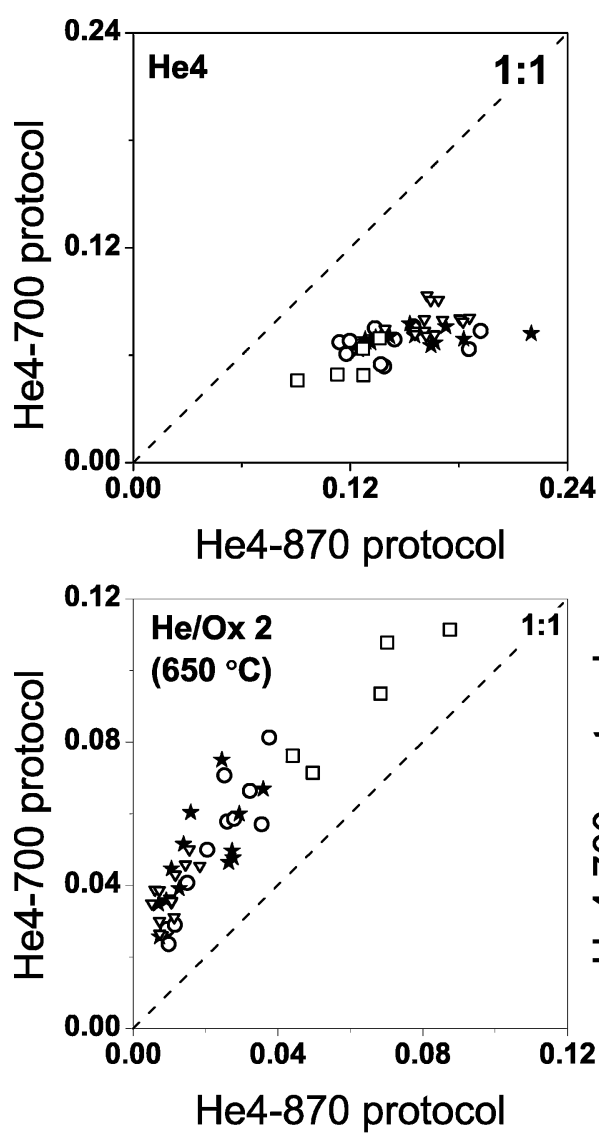

- Denuded 24-h

$\star$ Bare (undenuded) 24-h

$\nabla$ Bare 8-h

Bare 48-h and 96-h

\section{.}
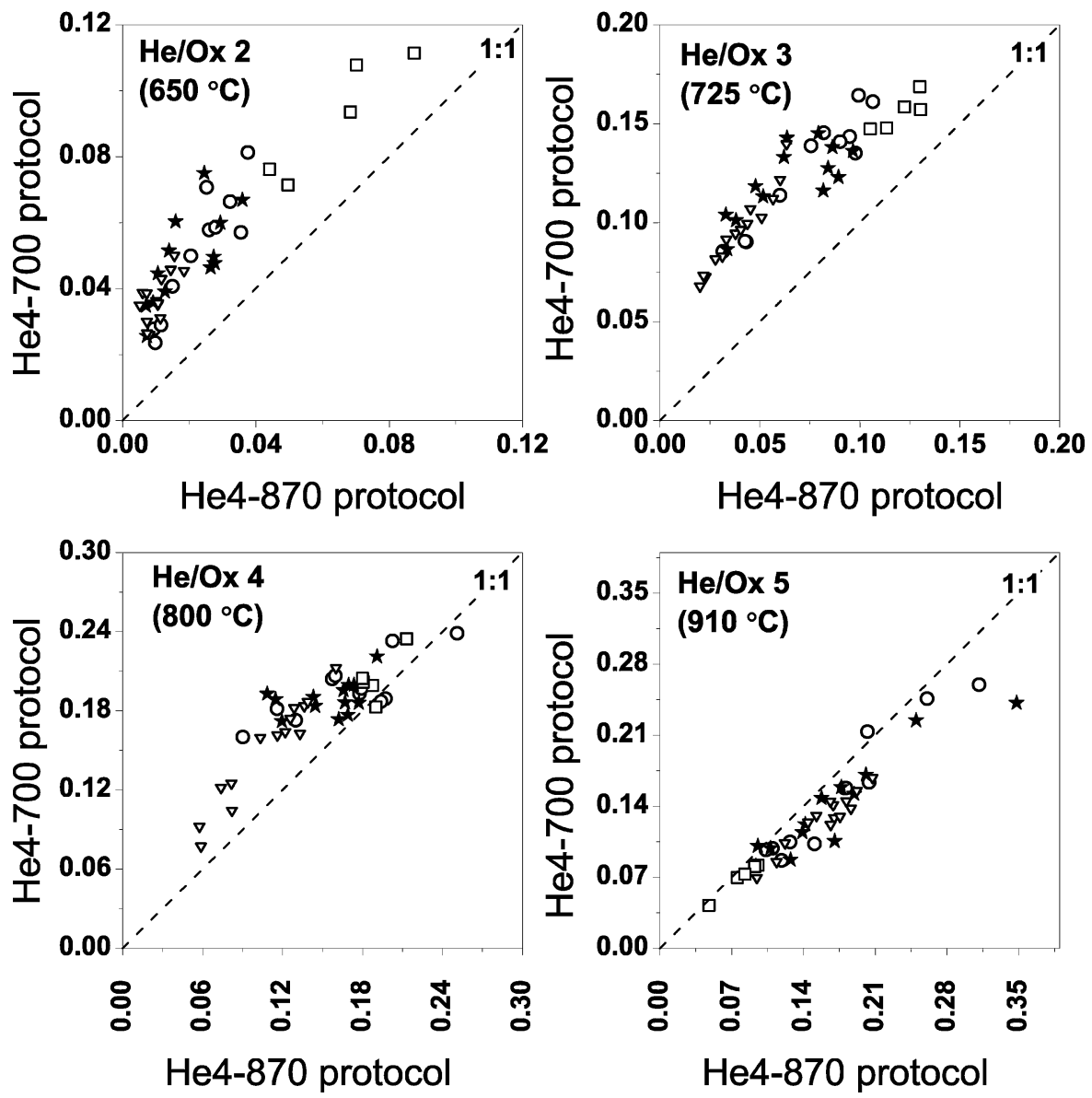

FIG. 5. Scatter plot of carbon peaks (as a fraction of total carbon) for paired filter punches analyzed with both the He4-700 and He4-870 protocols. Lowering the peak He-mode temperature from $870^{\circ} \mathrm{C}$ to $700^{\circ} \mathrm{C}$ shifts carbon from the $\mathrm{He} 4$ step to the lower-temperature steps of the He/Ox mode.

but does not eliminate, PC formation. For example, solvent extraction reduces the dip in laser attenuation associated with PC formation by $88 \%$ for the denuded sample shown in Figure 7 a and by $72 \%$ for the undenuded sample shown in Figure $7 \mathrm{~b}$ compared to the analyses of the untreated punches. Figure $7 \mathrm{~b}$ also shows that the sample transmission increases in the Heliummode as early as the He-3 step, which could be loss of PC (as only $72 \%$ of the pyrolysis is eliminated), or even some EC.

Loss of EC during solvent extraction is a concern. The optical EC loading on the extracted and untreated punches, as indicated by the net change in laser attenuation from the beginning to the end of the analysis, was almost the same (the ratio of net laser attenuation values being $97 \pm 8 \%$ for 14 

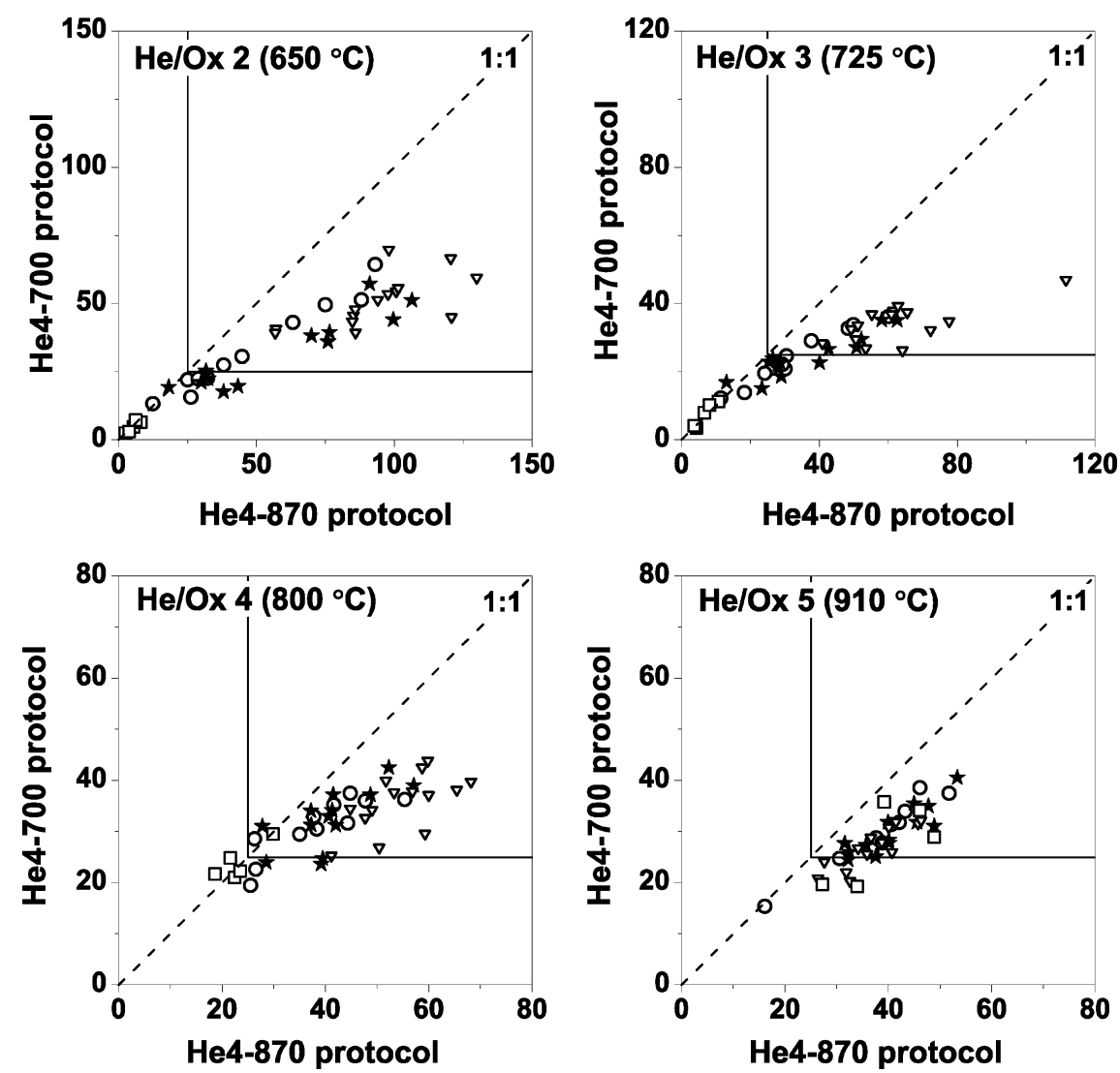

FIG. 6. Scatter plot of the specific attenuation $\left(\mathrm{m}^{2} / \mathrm{g}-\mathrm{C}\right)$ of each carbon peak for paired filter punches analyzed with both the He4-700 and He4-870 protocols. The mixture of EC and PC co-evolving in the He/Ox mode of the He4-870 protocol is more light-absorbing than the carbon coming off the filter in the He/Ox mode of the He4-700 protocol. The solid lines indicate the upper limit for $k_{\mathrm{EC}}\left(25 \mathrm{~m}^{2} / \mathrm{g}-\mathrm{C}\right)$; He4-870 samples to the left of and He4-700 samples below these lines have attenuation coefficients less than this upper bound. Symbols represent the same samples as in Figure 5.

extracted/untreated sample pairs). This indicates that there is minimal loss of EC during the solvent extraction procedure, and differences in carbon loading between the extracted and untreated punches can be attributed to removal of OC. Most of the carbon that evolves from the He/Ox mode of the analysis of the extracted punches is EC and increases in laser transmission during the analysis of extracted punches are primarily due to EC evolving from the sample.

\section{EVOLUTION OF PC AND EC}

A comparison of the carbon evolution from the paired solvent extracted and untreated punches provides strong evidence that PC and EC co-evolve and that this assumption is not valid for the Pittsburgh samples. Figure 7 shows that significantly less carbon evolves from the extracted punch than from the untreated punch in the He/Ox-mode of both protocols (He4-870 and He4-700). Since the extraction procedure did not affect the EC loading, the $\mathrm{He} / \mathrm{Ox}$ carbon removed by the solvent-extraction is PC. Notably, the He/Ox carbon removed by extraction does not just evolve at the beginning of $\mathrm{He} / \mathrm{Ox}$ mode, but throughout the entire mode providing strong evidence that PC and EC co-evolve in both protocols.
The transmission-defined OC/EC split of the solventextracted punches always occurs earlier in the analysis cycle compared to the analysis of untreated punches. For such a shift to occur, some fraction of the native EC must evolve before the transmission-defined OC/EC split of the untreated punch. We refer to this as "pre-split EC." The grey area shown in Figure 7 is an estimate of the pre-split EC; this area is the carbon that evolves from the extracted punch, between the transmissiondefined OC/EC splits of the extracted and untreated punches. This estimate represents an upper-bound for the pre-split EC because the solvent-extraction procedure does not completely eliminate PC formation. For the sample shown in Figure 7a analyzed with the He4-870 protocol, up to $50 \%$ of the native EC evolved before the transmission-defined OC/EC split of the untreated punch. In addition, a large fraction of this pre-split EC evolved in the He4 step. There is less pre-split EC when samples are analyzed with the He4-700 protocol; for the sample shown in Figure 7b up to 35\% of native EC evolved before the transmission-defined OC/EC split of the untreated punch. Increasing the length of the He/Ox steps does not change the conclusion that some fraction of the native $\mathrm{EC}$ evolves before the transmission-defined OC/EC split. 

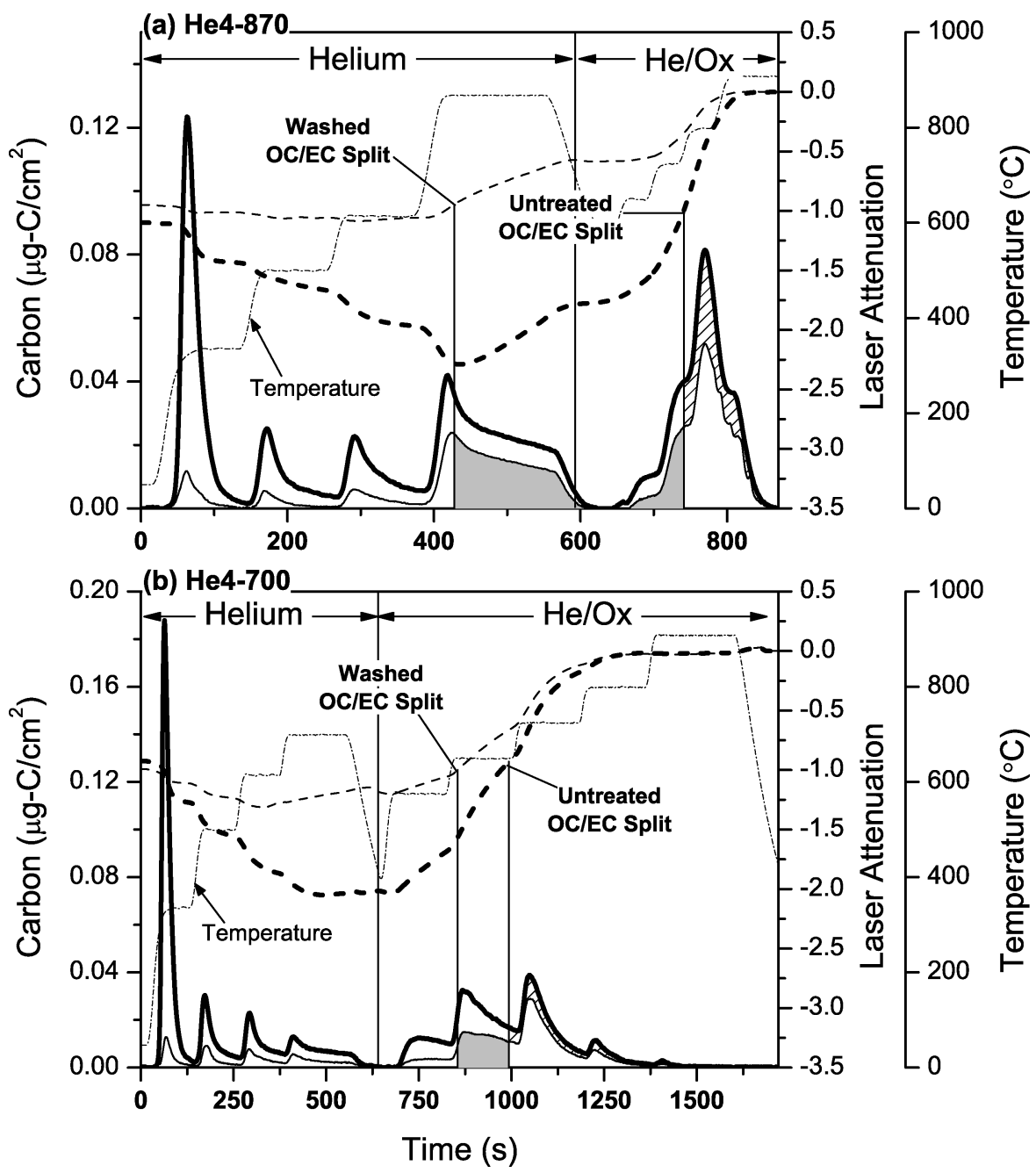

FIG. 7. Thermograms of untreated and solvent-extracted samples collected on April 17, 2002: (a) He4-870 analyses of a denuded sample; (b) He4-700 analyses of an undenuded sample using extended duration He/Ox peaks. The thin lines indicate solvent-extracted or washed punches and thick lines indicate the untreated punches. The solid lines are the carbon data plotted against the left axis, and the dashed lines are the laser attenuation data, $\ln \left(\mathrm{I} / \mathrm{I}_{\text {end }}\right)$, plotted against the right axis. EC and PC co-evolve for samples analyzed with both protocols. The grey area indicates carbon that evolves in the He/Ox mode from the solvent extracted punch before the OC/EC split of the untreated punch. This carbon is native-EC evolving before the OC/EC split. The hashed area is carbon removed by solvent extraction that evolves after the OC/EC split of the untreated punch. This carbon is PC evolving after the OC/EC split. The sample analyzed with the He4-870 protocol shows premature EC loss, causing the OC/EC split of the extracted punch to be defined in the He-mode.

Comparing the solvent-extracted and untreated punches one can also estimate the amount of PC that evolves after the transmission-defined OC/EC split of the untreated filter. For the samples shown in Figures 7a and 7b, solvent extraction removed approximately $30 \%$ of the He/Ox carbon after the transmissiondefined OC/EC split of the untreated filter. This carbon is indicated by the hashed area. It represents a lower-bound estimate of the PC that evolves after the split because solvent extraction did not eliminate $\mathrm{PC}$ formation.

Another issue is the type of light-absorbing carbon that evolves in the He-mode of the analysis-is it PC or native EC? Yu et al. (2002) suggest that it is PC. Figure 7 shows that some light-absorbing carbon evolves in the $\mathrm{He} 4$ temperature step from the solvent-extracted punches analyzed with both protocols. This loss is significantly larger for samples analyzed with the He4-870 protocol compared to those analyzed with the He4-700 protocol. For extracted samples analyzed with the He4-870 protocol (Figure 7a), the transmission-defined OC/EC split always occurs in He-mode, i.e., the sample transmission rises above the initial, pre-pyrolysis value in the He-mode. The only explanation is that native EC is clearly evolving in the $870^{\circ} \mathrm{C}$ step of the He-mode. Notably the split occurs very early in the He4 step, indicating the EC evolves throughout most of the step, making it difficult to simply shorten the He4 step to avoid the premature loss of EC. For solvent-extracted samples analyzed with the He4-700 protocol (Figure 7b), the transmission-defined OC/EC split always occurs in the He/Ox mode; therefore, the modest increase in laser transmission observed in the He-mode 
of extracted samples analyzed with the He4-700 protocol could be due to a minor loss of either native EC or PC. Given that PC and EC co-evolve through the analysis, our results do not contradict Yu et al.'s hypothesis, but complement it; the light-absorbing carbon evolving at the $\mathrm{He} 4 \mathrm{step}\left(870^{\circ} \mathrm{C}\right)$ of the He4-870 analysis of untreated samples is native EC, possibly combined with PC.

Analysis of extracted and untreated pairs of filter punches from fourteen denuded and undenuded samples taken over ten days provided results consistent with those shown in Figure 7. This means that PC and EC co-evolve in the He/Ox mode of the analysis for both protocols. In addition, although our discussion has focused on the He4-700 and He4-870 protocol, similar comparisons (as those shown in Figure 7) for punches analyzed with the He4-550 protocol also show clear evidence of co-evolution of PC and EC. Therefore, the first assumption underlying the thermal-optical analysis method that PC evolves before EC is not valid for the Pittsburgh samples. The co-evolution of PC and EC is consistent with recent results from $\mathrm{Yu}$ and co-workers (Yang and Yu 2002; Yu, Xu, and Yang 2002). Malm et al. (1994) also provides interesting evidence for the co-evolution of PC and $\mathrm{EC}$; in particular, their results suggest that native EC evolves in the lower temperature steps of the He/Ox mode. A consequence of this co-evolution is that it is incorrect to view the post-split carbon as native EC. At best, the mass of the post-split carbon will be equal to native EC. Whether or not this occurs depends on the optical properties of PC and EC, as discussed in the next section.

A second important conclusion from our analysis of the solvent-extracted punches is that increasing the peak He-mode temperature from $700^{\circ} \mathrm{C}$ to $870^{\circ} \mathrm{C}$ systematically shifts when native EC evolves from a sample. Notably, native EC is shifted from the low-temperature steps of the $\mathrm{He} / \mathrm{Ox}$ mode of the He4700 protocol to the He-mode in the He4-870 analysis. Figures 4(a) and 5 indicate that this shift is consistent across a large number of samples. An important consequence of this shift is that increasing the peak $\mathrm{He} 4$ temperature changes the relative rates at which $\mathrm{PC}$ and $\mathrm{EC}$ evolve from the filter. The native EC evolves earlier in the analysis cycle compared to PC when samples are analyzed with the He4-870 protocol. Again, this will only be a problem if PC and EC have different optical properties.

\section{ATTENUATION COEFFICIENTS OF PC AND EC}

The second assumption underlying thermal-optical OC/EC analysis is that $\mathrm{PC}$ and $\mathrm{EC}$ have the same optical properties. If sample transmission is used to define the OC/EC split this means that the specific attenuation coefficients of $\mathrm{PC}\left(k_{\mathrm{PC}}\right)$ and $\mathrm{EC}\left(k_{\mathrm{EC}}\right)$ should be the same. Estimates for $k_{\mathrm{PC}}$ and $k_{\mathrm{EC}}$ can be calculated by applying Equation (1) to the thermograms of the solventextracted and untreated filters. The calculations are based on the measured laser transmission at the beginning and the end of the $\mathrm{He} / \mathrm{Ox}$ mode and the amount of carbon that evolves in the $\mathrm{He} / \mathrm{Ox}$ mode. For the sample shown in Figure $7 \mathrm{a}$, the average attenu- ation coefficients of the $\mathrm{He} / \mathrm{Ox}$ carbon are $16 \mathrm{~m}^{2} / \mathrm{g}-\mathrm{C}$ for the solvent-extracted punch and $31 \mathrm{~m}^{2} / \mathrm{g}-\mathrm{C}$ for the untreated punch. The He/Ox carbon on the solvent-extracted punch is mainly $\mathrm{EC}$, and so we assume $k_{\mathrm{EC}}$ for this sample as $16 \mathrm{~m}^{2} / \mathrm{g}-\mathrm{C}$. The $\mathrm{He} / \mathrm{Ox}$ carbon on the untreated punch is a mixture of PC and EC; therefore $k_{\mathrm{PC}}$ must be greater than $k_{\mathrm{EC}}$. The specific attenuation coefficient of the carbon removed by solvent extraction provides an estimate for $k_{\mathrm{PC}}$. This value can be derived by combining the results from the analysis of the untreated and solvent-extracted punches, and applying Equation (1). This approach yields a $k_{\mathrm{PC}}$ value of $56 \mathrm{~m}^{2} / \mathrm{g}-\mathrm{C}$ for the sample shown in Figure $7 \mathrm{a}$.

To develop a more robust estimate for $k_{\mathrm{EC}}$ and $k_{\mathrm{PC}}$, we repeated the extraction procedure on more ambient samples. A challenge is that the extraction procedure does not eliminate PC formation and therefore does not cleanly separate PC and EC. For six ambient samples collected on different days in which the extraction procedure reduced PC formation by over $70 \%$ (average reduction $81 \%$ ), the specific attenuation of the He/Ox carbon evolving in the solvent-extracted filter, $k_{\mathrm{EC}}$, was $22.9 \pm 5.4 \mathrm{~m}^{2} / \mathrm{g}$ $\mathrm{C}$ (average \pm standard deviation). The average attenuation coefficient of the He/Ox carbon removed by solvent extraction, $k_{\mathrm{PC}}$, was $35.2 \pm 12.7 \mathrm{~m}^{2} / \mathrm{g}-\mathrm{C}$. Therefore, $k_{\mathrm{PC}}$ is consistently higher than $k_{\mathrm{EC}}$, but the large standard deviation suggests significant sample-to-sample variability in $k_{\mathrm{PC}}$. Some of this variability may also be due to changes in extraction efficiency. In addition, systematic differences were observed in the $k_{\mathrm{PC}}$ values for samples analyzed with the different protocols. The $k_{\mathrm{PC}}$ values of samples analyzed with the He4-870 protocol were on average around $40 \%$ greater than those analyzed with the He4-700 protocol. This difference may indicate additional formation and/or darkening of PC at higher temperatures in the He-mode. Finally, these values of $k_{\mathrm{PC}}$ may be at the lower end of the possible range, since for five of the six samples, pyrolysis darkens or reduces the transmission of the untreated samples close to or beyond the limits of linear laser response (as per Gundel et al. [1984], $\ln \left(I_{\text {blank }} / I_{\text {sample }}\right)$ of 2.0) where the "shadowing" or "light-pipe" effect is seen.

Our estimates of $k_{\mathrm{EC}}$ are consistent with literature values for EC deposited on quartz filters, which range from $8.1 \mathrm{~m}^{2} / \mathrm{g}$ to $25.4 \mathrm{~m}^{2} / \mathrm{g}$ for solvent-extracted ambient filters (Gundel et al. 1984; Petzold, Kopp, and Niessner 1997). In comparing different values, one must always keep in mind that $k_{\mathrm{EC}}$ depends on various factors including aging, wavelength, the extraction/thermal methods, and the filter substrate. For example, Bond and Bergstrom (2006) suggests a mass absorption coefficient of $7.5 \pm 1.2 \mathrm{~m}^{2} / \mathrm{g}$ for fresh light absorbing carbon, measured at $550 \mathrm{~nm}$; assuming absorption varies inversely with wavelength gives $6 \mathrm{~m}^{2} / \mathrm{g}-\mathrm{C}$ at $680 \mathrm{~nm}$ (the wavelength of the laser in our Sunset instrument). The aerosol in Pittsburgh is highly aged; aging tends to increase the absorption coefficient of EC due to coating of the EC by non-absorbing species (Fuller, Malm, and Kreidenweis 1999). Finally, collection of EC on a quartz filter enhances the absorption coefficient by about a factor of two (Weingartner et al. 2003). However, although there appears to be 

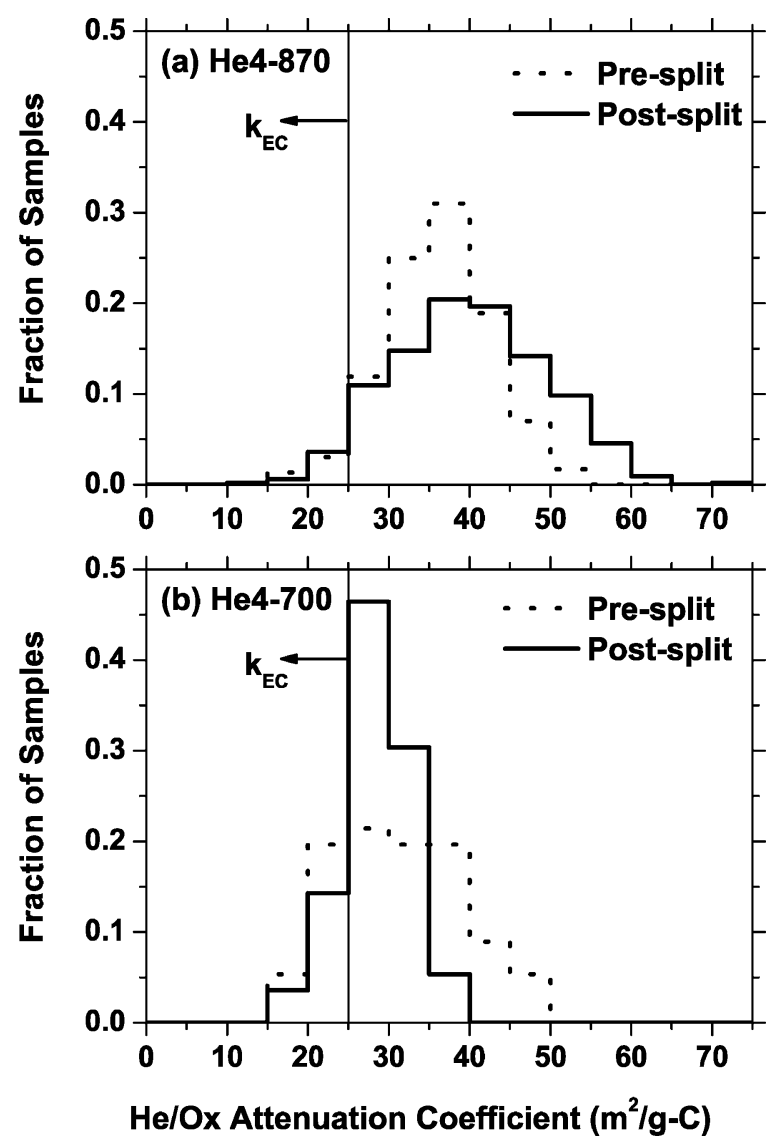

FIG. 8. Histograms of specific attenuation coefficient of pre- and post-split $\mathrm{He} / \mathrm{Ox}$ carbon measured using the (a) He4-870 protocol (number of samples, $\mathrm{N}=529)$, and (b) He4-700 protocol $(\mathrm{N}=56)$. The results are based on the analysis of 24-h denuded and undenuded samples. The vertical line indicates the upper bound for $k_{\mathrm{EC}}\left(25 \mathrm{~m}^{2} / \mathrm{g}-\mathrm{C}\right)$.

no unique value for $k_{\mathrm{EC}}, 25 \mathrm{~m}^{2} / \mathrm{g}-\mathrm{C}$ appears to be a reasonable upper bound (Liousse, Cachier and Jennings 1993). Significantly less research has been published about $k_{\mathrm{PC}}$; Chow et al. (2004) report $k_{\mathrm{PC}}$ values greater than $45 \mathrm{~m}^{2} / \mathrm{g}-\mathrm{C}$.

Additional insight into $k_{\mathrm{EC}}$ and $k_{\mathrm{PC}}$ can be gained by examining histograms of attenuation coefficients of pre- and post-split $\mathrm{He} / \mathrm{Ox}$ carbon for samples analyzed with the He4-700 and He4870 protocols, shown in Figure 8. For the vast majority of the samples, the $\mathrm{He} / \mathrm{Ox}$ carbon attenuation coefficient is greater than $25 \mathrm{~m}^{2} / \mathrm{g}-\mathrm{C}$. This indicates that $k_{\mathrm{PC}}$ is almost always greater than $k_{\mathrm{EC}}$ (assuming that $25 \mathrm{~m}^{2} / \mathrm{g}-\mathrm{C}$ is the upper limit for $k_{\mathrm{EC}}$ ). The $\mathrm{He} / \mathrm{Ox}$ carbon measured with the He4-870 protocol is significantly darker than that measured with the He4-700 protocol. This is consistent with premature evolution of EC in the Hemode of the He4-870 protocol, which means PC contributes a much larger fraction of the He/Ox carbon of samples analyzed with the He4-870 protocol than during analysis with the He4700 protocol. An additional factor is that $k_{\mathrm{PC}}$ values for samples analyzed with the He4-870 protocol are larger than those of samples analyzed with the He4-700 protocol as noted earlier.
By comparing the average post-split carbon attenuation coefficients of the two protocols ( 36 and $28 \mathrm{~m}^{2} / \mathrm{g}-\mathrm{C}$ for the He $4-870$ and He4-700 protocols, respectively), one can estimate the average bias in the TOT-EC defined by the two protocols. The difference in these values means that if the net laser attenuation of the two samples is the same, on average $29 \%$ more carbon evolves after the split (i.e., $29 \%$ more TOT-EC) for a sample analyzed with the He4-700 protocol compared to an analysis with the He4-870 protocol. A $29 \%$ bias is consistent with the intercomparison results shown in Figure 3, which is based on a small subset of the samples shown in Figure 8.

Our examination of the attenuation coefficients of the solventextracted and untreated samples indicates that PC has a significantly higher attenuation coefficient than $\mathrm{EC}\left(k_{\mathrm{PC}}>k_{\mathrm{EC}}\right)$. Therefore the second assumption underlying the thermal optical technique is also not valid. This means that the post-split carbon is not equal to the native EC mass. The bias will depend on the attenuation coefficient of the post-split carbon relative to that of the native EC. For the Pittsburgh samples, $k_{\mathrm{PC}}$ is greater than $k_{\mathrm{EC}}$; combined with co-evolution of $\mathrm{PC}$ and $\mathrm{EC}$, this means the TOT-defined EC is likely biased low compared to the true EC. The extent of this bias depends on the relative rate at which PC and $\mathrm{EC}$ evolve off the filter. An additional complication is that there is no unique value for $k_{\mathrm{EC}}$ or $k_{\mathrm{PC}}$, since optical properties depend on aerosol composition, measuring wavelength, and other parameters.

\section{Effect of Changes in the Relative Rates of EC and PC Evolution on the OC/EC Split}

We now use a model based on the Lambert-Beer law to illustrate quantitatively how changing the relative rate at which EC and $\mathrm{PC}$ evolve from the filter biases the transmission-defined OC/EC split. The model assumes that only two types of carbon, PC and EC, evolve from the sample after laser transmission reaches its minimum value. Under these conditions, the change in laser transmission (once it has passed its nadir) can be defined as,

$$
I(t)=I(t-1) \cdot \exp \left[C(t) \cdot\left(f_{\mathrm{PC}}(t) k_{\mathrm{PC}}+f_{\mathrm{EC}}(t) k_{\mathrm{EC}}\right)\right]
$$

where $I(t-1)$ and $I(t)$ are the laser transmission values at the start and at the end of a discrete time step during which the carbon mass $C(t)$ has evolved from the sample, and $f_{\mathrm{PC}}(t)$ and $f_{\mathrm{EC}}(t)$ are the PC and EC fractions of $C(t)$, with $f_{\mathrm{PC}}(t)+f_{\mathrm{EC}}(t)=1$. The model can easily be generalized to consider additional types of carbon such as non-light-absorbing organic carbon slipping into the He/Ox mode, but two are sufficient to illustrate how changing the relative rate at which different types of carbon with different optical properties evolve changes the TOT-EC. We also assume that $k_{\mathrm{EC}}$ and $k_{\mathrm{PC}}$ are not temperature dependent, but this assumption could also be easily relaxed.

We apply the model using data from the sample shown in Figure 7(a), using the measured carbon evolution during each 
second of the analysis for $C(t)$, and the measured minimum laser transmission as a starting point. These values are from the OC/EC analysis of the untreated punch. The native EC is assumed to be the carbon evolving from the solvent-extracted punch after the minimum laser signal of the untreated sample, $5.6 \mu \mathrm{g}-\mathrm{C} / \mathrm{cm}^{2}$. We assume that PC is the difference of the carbon combusting in the He/Ox mode of the two analyses, $2.2 \mu \mathrm{g}$ $\mathrm{C} / \mathrm{cm}^{2}$, likely an under-estimate since we do not include any PC that evolves in the He-mode (though it could also contain some EC lost from the solvent-extracted sample). We use the specific attenuation coefficient of the He/Ox carbon of the solventextracted sample as $k_{\mathrm{EC}}\left(16 \mathrm{~m}^{2} / \mathrm{g}-\mathrm{C}\right)$, and the specific attenuation coefficient of the carbon removed from the He/Ox mode of the analysis for $k_{\mathrm{PC}}\left(56 \mathrm{~m}^{2} / \mathrm{g}-\mathrm{C}\right)$.

Using these inputs, Figure 9 shows model calculations for two scenarios: (a) "PC-first," the darker PC evolves completely before the EC; and (b) "EC-first," the EC completely evolves before the PC. These scenarios define the minimum and maximum amounts of TOT-EC. Underestimation of PC coupled with some apparent loss of EC in the solvent-extracted sample (indicated by the different net laser attenuation for the untreated and solvent-extracted punches) causes the computed final laser transmission to be slightly less than the measured final value.

The model calculations clearly illustrate how changing the relative rate at which $\mathrm{PC}$ and $\mathrm{EC}$ evolve from the filter alters the OC/EC split. Since $k_{\mathrm{PC}}$ is greater than $k_{\mathrm{EC}}$, the first scenario ("PC-first") yields the maximum amount of TOT-EC. This scenario is consistent with the assumptions underlying the thermal-optical method; therefore it yields a TOT-EC of $5.7 \mu \mathrm{g} / \mathrm{cm}^{2}$, in good agreement with the native EC used as an input for the calculations (a little higher since the split is calculated at $97 \%$ of the initial laser value). The second scenario, "EC-first," defines the minimum value for TOT-EC at $1.7 \mu \mathrm{g} / \mathrm{cm}^{2}$, a factor of three smaller than the native EC. The large difference between these two limits is due to the significantly different attenuation coefficients for PC and EC. Although this second scenario is unlikely to occur during an actual analysis, it provides a useful reference point.

The relative rate at which $\mathrm{PC}$ and $\mathrm{EC}$ evolve (with respect to each other) shifts the OC/EC split between the two limits defined by the model. More premature evolution of EC, the farther the split shifts towards the EC-first limit. Figure 9 shows that the actual analysis of the untreated filter using the He4870 protocol falls between the two calculated limits. This is expected given the co-evolution of PC and EC, especially the significant premature evolution of EC in the $\mathrm{He} 4$ step of the $\mathrm{He} 4-$ 870 protocol. The TOT-EC measured by the He4-870 protocol is $4.4 \mu \mathrm{g}-\mathrm{C} / \mathrm{cm}^{2}$, about $20 \%$ smaller than the value determined by solvent extraction $\left(5.6 \mu \mathrm{g}-\mathrm{C} / \mathrm{cm}^{2}\right)$.

The results illustrated in Figure 9 provide a framework for understanding the systematic biases between the TOT-EC values measured by different temperature protocols. For samples analyzed with the He4-870 protocol, a larger fraction of the EC evolves earlier in the analysis cycle-in the $\mathrm{He} 4$ temperature step-compared to samples analyzed with the He4-700 protocol. This shift alters the TOT-EC because the attenuation coefficients

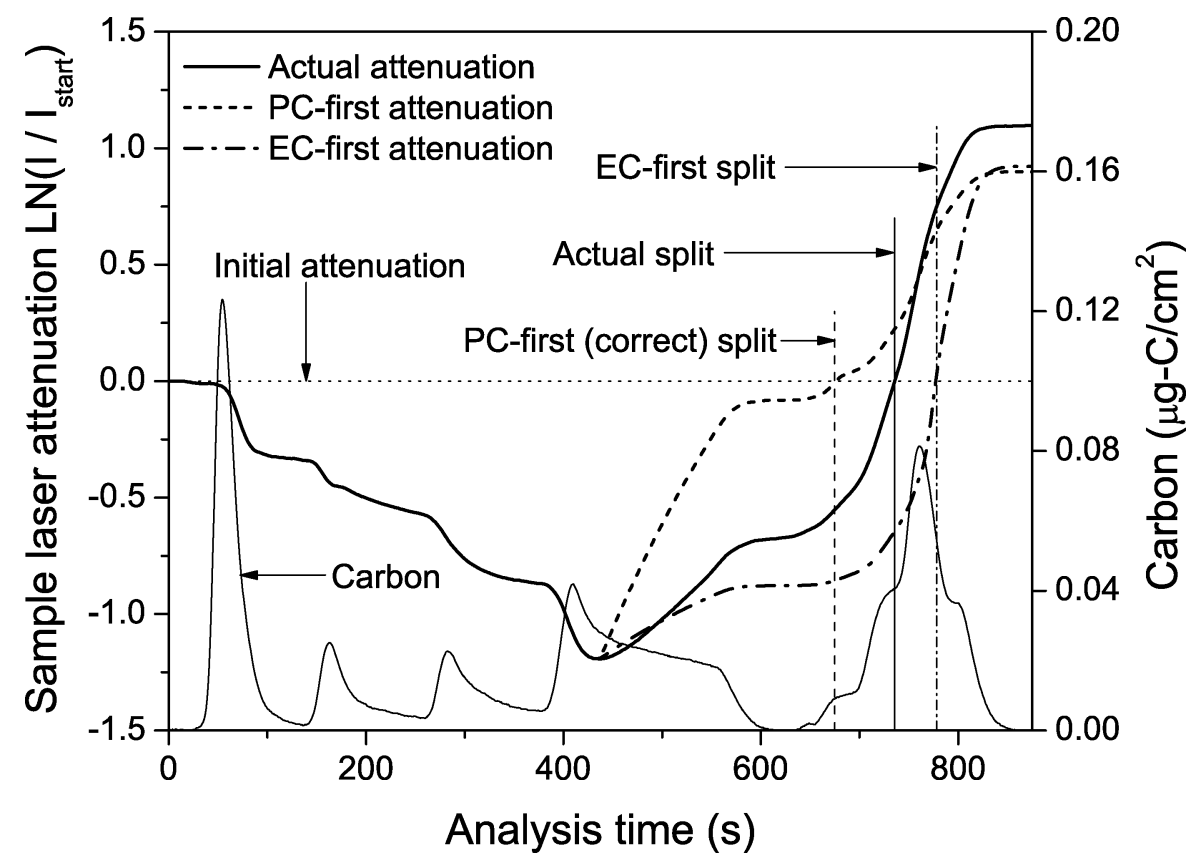

FIG. 9. Predicted change in laser attenuation due to evolution of light-absorbing carbon (PC and EC) off the filter for the sample shown in Figure 7a. The PC-first scenario satisfies the method assumptions, and is the true OC/EC split. Due to the higher specific attenuation coefficient of PC $\left(k_{\mathrm{PC}}>k_{\mathrm{EC}}\right.$ ), the rise in laser transmission in the PC-first scenario is much sharper than is actually observed. The EC-first model is an extreme case which would give the maximum underestimation of EC. In reality, EC and PC co-evolve, and the observed laser transmission falls in between the two extremes. 
of EC and PC are different. In Pittsburgh, $k_{\mathrm{PC}}$ is greater than $k_{\mathrm{EC}}$, so shifting EC evolution to earlier in the analysis cycle (relative to PC) reduces the amount of TOT-EC. Therefore, at least for samples collected in Pittsburgh, the premature evolution of EC causes the TOT-EC defined by the He4-870 protocol to consistently underestimate the native EC.

\section{Peak He-mode Temperature and Non-Light-Absorbing Carbon}

Lowering the peak He-mode temperature to $700^{\circ} \mathrm{C}$ improves the OC/EC split by avoiding premature evolution of EC, and so the question arises: would lowering the peak He-mode temperature further, say to $550^{\circ} \mathrm{C}$ as in the IMPROVE protocol (Chow et al. 1993), give better answers for the OC/EC split? A concern created by lowering the peak He-mode temperature is that not all of the $\mathrm{OC}$ will volatilize or pyrolyze during the inert portion of the analysis resulting in non-light-absorbing carbon evolving in the He/Ox mode (Novakov and Corrigan 1995; Schauer et al. 2003). To examine issues associated with non-light-absorbing carbon we analyzed quartz filters loaded with either levoglucosan (99+\% pure; ACROS Organics) or secondary organic aerosol (SOA). Levoglucosan, a pyrolysis product of cellulose, is a major component of wood smoke (Simoneit et al. 1999; Schauer et al. 2001). The secondary organic aerosol (SOA) was created by reacting $\alpha$-pinene with ozone in the Carnegie Mellon University smog chamber.

Figure 10 shows thermograms of pre-baked blank quartz filters doped with about $10 \mu \mathrm{g}-\mathrm{C} / \mathrm{cm}^{2}$ of levoglucosan analyzed with the He4-700 (Figure 10a) and He4-550 (Figure 10b) protocols. These samples do not contain EC, so all of the He/Ox carbon is either OC or PC. Twenty-three percent of the carbon from the levoglucosan evolves in the He/Ox mode of the He4550 analysis versus only 5\% for the He4-700 analyses. Notably, a significant amount of the carbon evolves in the $615^{\circ} \mathrm{C}$ and $700^{\circ} \mathrm{C}$ steps of the He-mode of the He4-700 protocol. The laser data indicates modest formation of light-absorbing PC during the analysis of levoglucosan-doped filters, with the overall pyrolytic attenuation being a few percent of that typically observed on similarly loaded ambient samples. For analyses using the He4-550 protocol, the average attenuation coefficient of the $\mathrm{He} / \mathrm{Ox}$ carbon was very low, $1.2 \mathrm{~m}^{2} / \mathrm{g}-\mathrm{C}$, which indicates that the bulk of the $\mathrm{He} / \mathrm{Ox}$ carbon is non-light-absorbing. In contrast, the $\mathrm{He} / \mathrm{Ox}$ carbon evolving during analysis with the He4-700 protocol

(a) Levoglucosan analyzed with He4-700

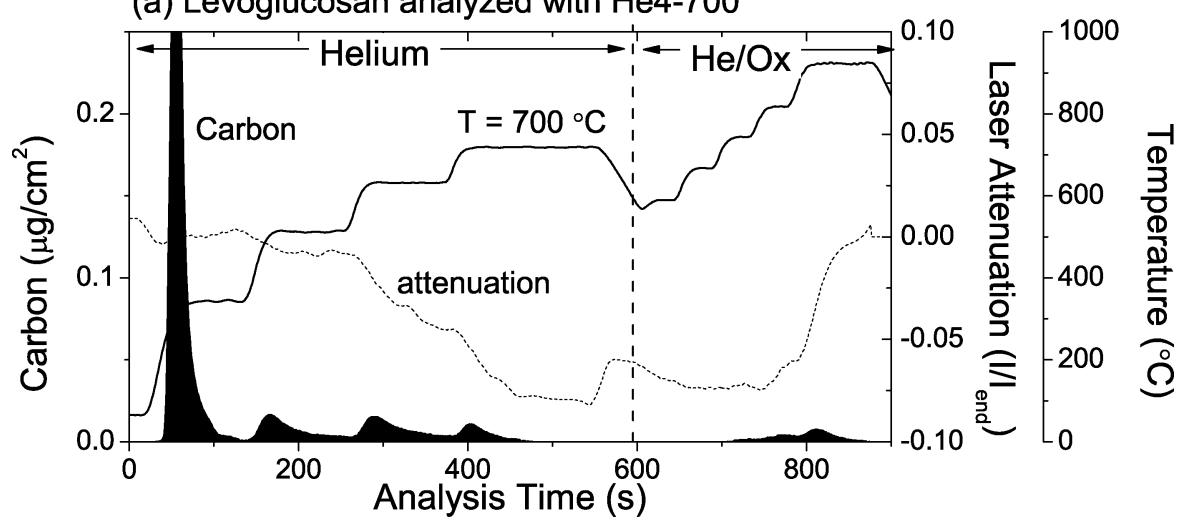

(b) Levoglucosan analyzed with He4-550

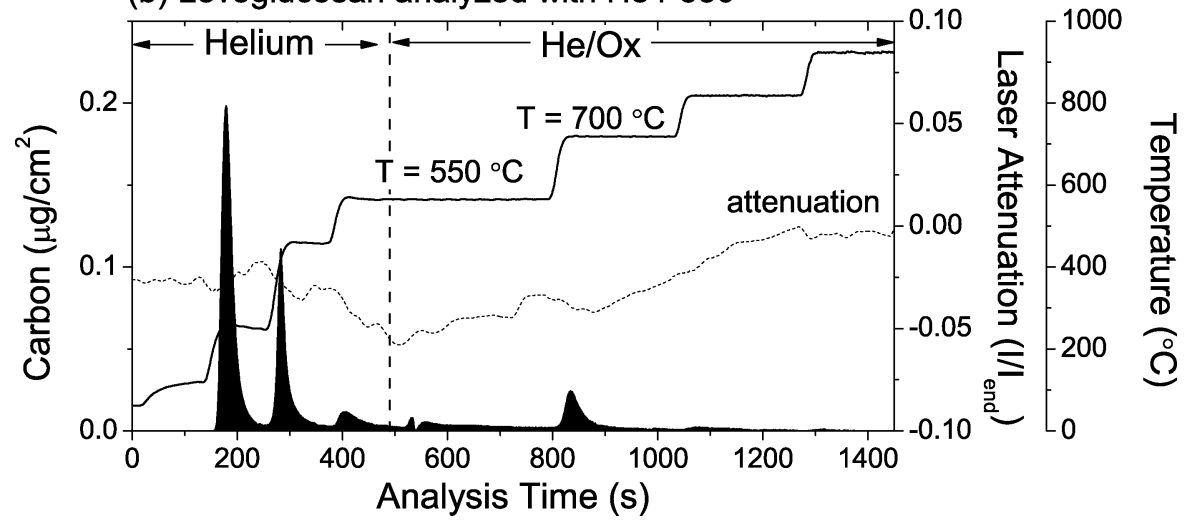

FIG. 10. Thermograms for levoglucosan-doped quartz filters analyzed using the (a) He4-700 and (b) the He4-550 protocols. 23\% of the levoglucosan carbon evolves in the He/Ox mode during analysis with the He4-550 protocol. As discussed in the text, this carbon is largely transparent (not light absorbing). In contrast, just $5 \%$ of the levoglucosan carbon evolves in the He/Ox mode during analysis with the He4-700 protocol. The laser dip due to pyrolysis in these samples is just a few percent of that seen with ambient samples (e.g., Figure 1). 

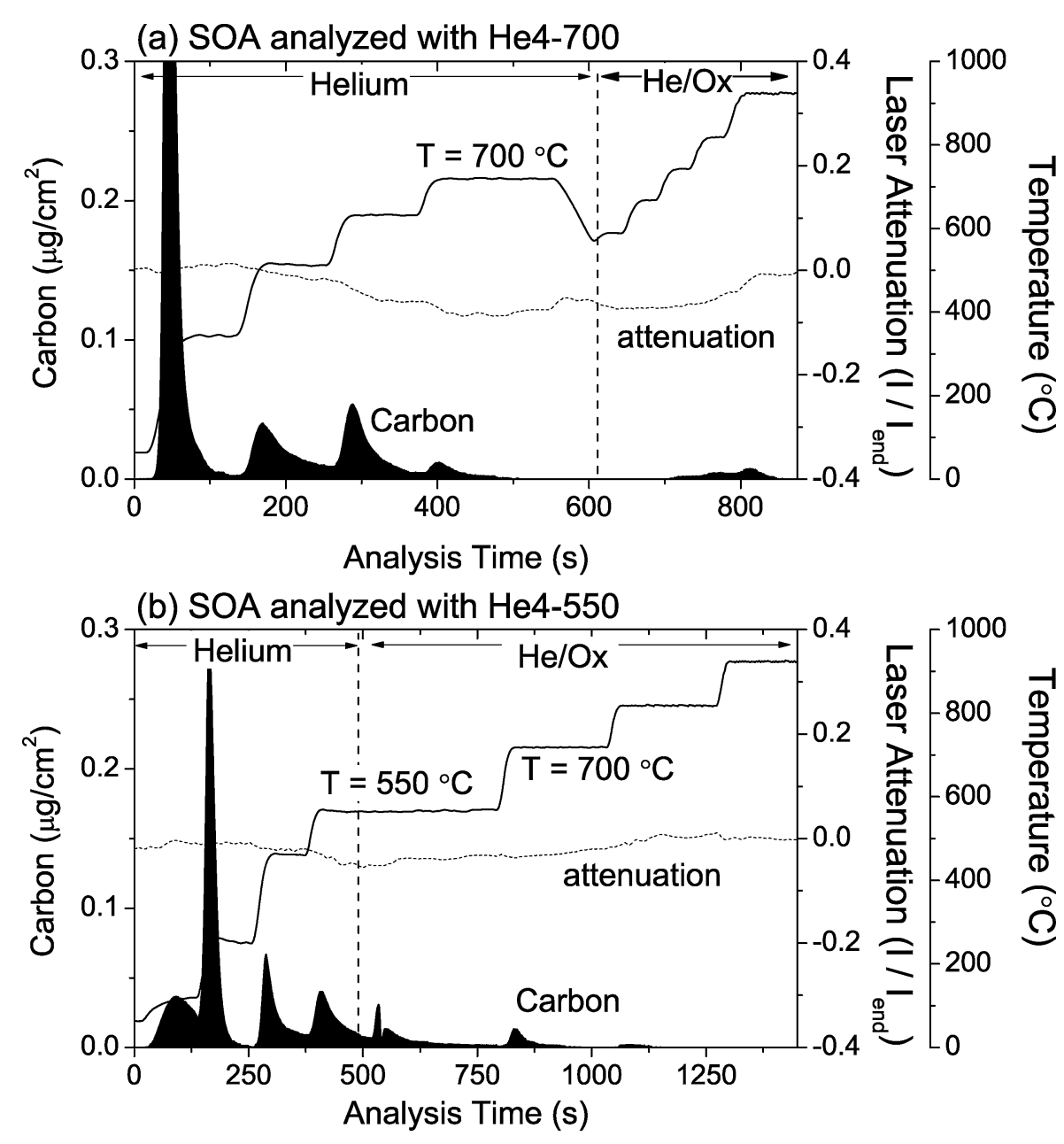

FIG. 11. Thermograms for secondary organic aerosol (SOA) collected on quartz filters analyzed using the (a) He4-700 and (b) the He4-550 protocols. The SOA was formed by the reaction of $\alpha$-pinene with ozone in the Carnegie Mellon University smog chamber.

absorbs light, with an average attenuation coefficient of $18 \mathrm{~m}^{2} / \mathrm{g}-\mathrm{C}$.

Thermograms of SOA analyzed with both the He4-700 protocol and He4-550 protocols are shown in Figure 11. The results for SOA are similar to those of levoglucosan-doped filters. First, some fraction of the SOA carbon evolves in the He/Ox mode with both protocols: $12 \%$ for the $\mathrm{He} 4-550$ protocol versus $5 \%$ for the He4-700 protocol. Minor amounts of PC formation are apparent in the SOA analyses using both protocols; the average attenuation coefficient of the He/Ox carbon evolving from the He4-550 protocol is $2.2 \mathrm{~m}^{2} / \mathrm{g}-\mathrm{C}$ versus $10.5 \mathrm{~m}^{2} / \mathrm{g}$-C for the He4-700 protocol. Finally, a significant fraction of the He/Ox carbon during the SOA analysis evolves in the higher temperature He/Ox steps in both protocols. For the He4-550 protocol, one-third of the $\mathrm{He} / \mathrm{Ox}$ carbon evolved at $700^{\circ} \mathrm{C}$ or higher. Overall, however, the problems associated with SOA appear to be less severe than those associated with levoglucosan for the He4-550 protocol.

Although Figures 10 and 11 show that significant amounts of relatively non-light-absorbing carbon evolve in the $\mathrm{He} / \mathrm{Ox}$ mode during the analysis of levoglucosan and SOA with the He4-550 protocol, this carbon is not misclassified as EC. So, for these simple systems, the transmission defined OC/EC split works. A problem arises only if the non-light-absorbing organics evolve off the filter after the OC/EC split. With ambient samples, which are a complex mixture of many different organics and EC, we cannot isolate this effect.

To illustrate the potential bias created by the slip of nonlight-absorbing carbon on the TOT-EC, Figure 12 presents thermograms from as-collected tunnel samples superimposed on thermograms of levoglucosan-doped tunnel samples, analyzed with both the He4-700 (Figure 12a) and He4-550 (Figure 12b) protocols. Figure 12a shows that negligible levoglucosan carbon evolves in the He/Ox mode of the samples analyzed with the He4-700 protocol. Consequently, the measured TOT-EC remains relatively unchanged despite the addition of levoglucosan (4.9 $\mu \mathrm{g}-\mathrm{C} / \mathrm{cm}^{2}$ for the as-collected tunnel sample compared to $5.1 \mu \mathrm{g}-\mathrm{C} / \mathrm{cm}^{2}$ for the doped sample). However, adding levoglucosan increases the TOT-EC of the tunnel sample analyzed with the He4-550 protocol, from $5.5 \mu \mathrm{g}-\mathrm{C} / \mathrm{cm}^{2}$ for the as-is tunnel sample to $6.5 \mu \mathrm{g}-\mathrm{C} / \mathrm{cm}^{2}$ for the levoglucosan-doped sample. The 
(a) Levoglucosan-doped tunnel sample: He4-700

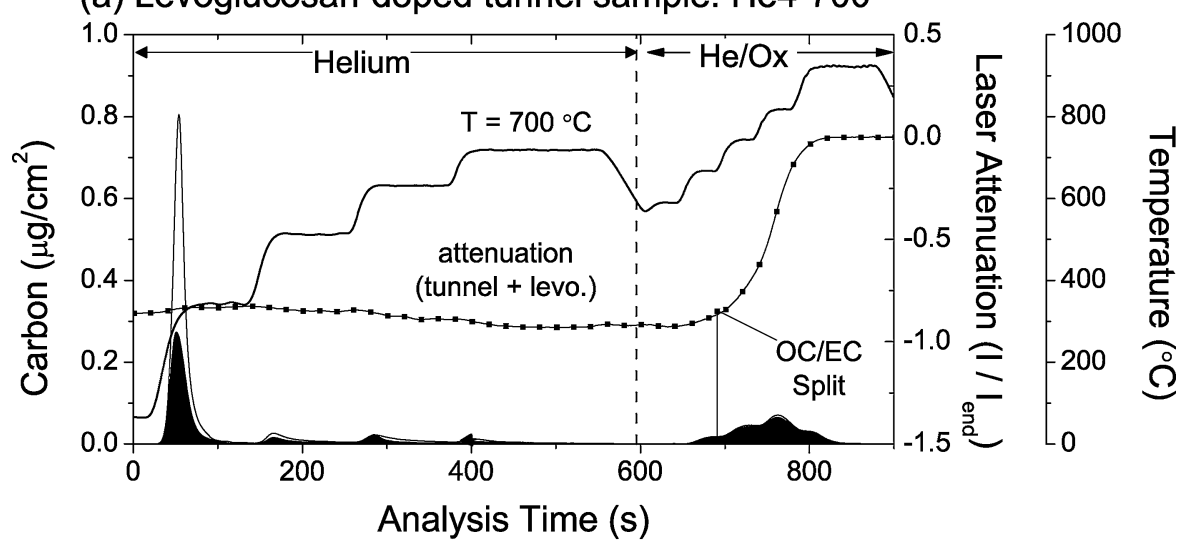

(b) Levoglucosan-doped tunnel sample: He4-550

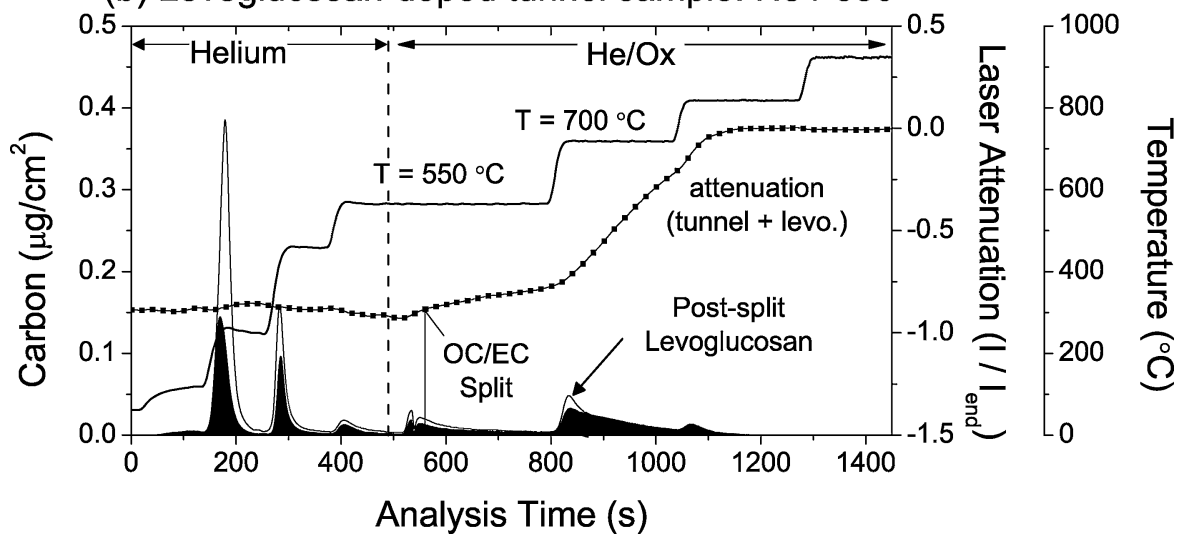

FIG. 12. Thermograms showing the effect of slip of non-light absorbing carbon in the He/Ox mode. Levoglucosan is spiked onto vehicular exhaust-dominated tunnel samples, and analyzed with the (a) He4-700 and (b) He4-550 protocols. As Figure 10 suggests, the He4-700 protocol does not experience significant slip of levoglucosan into the He/Ox mode, while during the He4-550 analysis, a significant fraction of the He/Ox mode levoglucosan comes off at the higher temperature steps, causing a positive bias in EC measurement with the He4-550 protocol relative to the undoped tunnel sample.

attenuation coefficient of the post-split carbon in the He4-550 protocol decreases from $16.9 \mathrm{~m}^{2} / \mathrm{g}-\mathrm{C}$ for the as-is tunnel sample to $13.6 \mathrm{~m}^{2} / \mathrm{g}-\mathrm{C}$ for the doped sample reflecting the effect of non-light-absorbing carbon on the net attenuation coefficient of the carbon evolving after the OC/EC split.

Figure $12 \mathrm{~b}$ provides evidence that other components of the aerosol affect the evolution of OC from the filter. For a clean quartz filter doped with levoglucosan, $23 \%$ of the levoglucosan OC came off in the He/Ox mode (Figure 10b) compared to only $14 \%$ of the levoglucosan OC coming off in the He/Ox mode of the levoglucosan-doped tunnel sample (Figure 12b). This indicates that ambient PM and $\mathrm{OC}$, which are mixtures of emissions from different sources, likely behave differently from pure compounds; results from Yu et al. (2002) also illustrate this effect.

We also compared EC measurements made on parallel punches taken from fourteen 24-hour ambient samples, using the He4-550 and He4-700 protocols. Six of the samples are high-wood-smoke samples (selected based on parallel levoglucosan concentrations). The remaining samples are from periods with low levoglucosan concentrations and moderate to high
OC/EC ratios, which suggest low wood smoke and some SOA. Total carbon and net attenuation for the paired punches were within $10 \%$, indicating that differences between the He4-550 and He4-700 analyses were not due to inhomogeneous filter loadings. Figure 13 compares the EC measurements made using the He4-700 and He4-550 protocols, as a fraction of the total carbon. For the six high wood smoke samples, the EC/TC ratio as per the He4-550 protocol is $1.52 \pm 0.15$ (average \pm standard deviation) times higher than the EC/TC ratio measured with the He4-700 protocol. A smaller, more variable bias is observed for the eight non-wood-smoke samples, for which the $\mathrm{He} 4-550 \mathrm{EC} / \mathrm{TC}$ ratio is $1.21 \pm 0.27$ times He4-700 EC/TC value. For three of the non-wood-smoke samples the agreement between the two protocols is within measurement uncertainty. The large variability in the bias between the two protocols for the non-wood-smoke samples is not unexpected given that the slip of non-light-absorbing carbon likely depends on sample composition and is not limited to wood smoke compounds. Such variability is consistent with the results of Schauer et al. (2003). Given the potential positive bias, it does not appear 


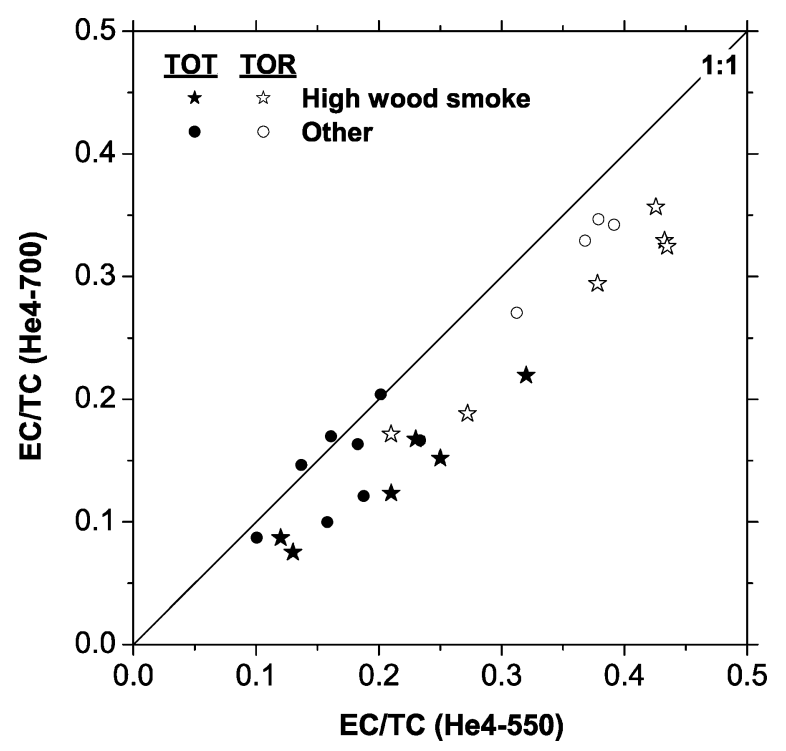

FIG. 13. Scatter plot of EC (as a fraction of TC) measured with He4-550 and He4-700 protocols for paired punches from high wood smoke and other ambient samples. The He4-550 protocol typically measures more EC than the He4-700 protocol, except for the three ambient samples close to the 1:1 line. Results are shown for OC/EC splits defined using transmission (TOT) and reflectance (TOR). TOR EC is not available for some of the samples included in the TOT inter-comparison, including the three TOT samples that show good agreement between the two temperature protocols.

advisable to reduce the peak He-mode temperature from $700^{\circ} \mathrm{C}$ to $550^{\circ} \mathrm{C}$.

\section{Reflectance versus Transmission}

Sample reflectance (TOR) is an alternative approach for defining the OC/EC split (Chow et al. 1993). Chow et al. (2004) found that TOR provides a more consistent OC/EC split than TOT across a wide variety of temperature protocols. Toward the end of this research the Carnegie Mellon University Sunset Laboratory OC/EC analyzer was upgraded to simultaneously measure both TOR and TOT EC. Figure 13 shows TOT- and TOR-EC measured for the set of ten samples used in the He4550 versus $\mathrm{He} 4-700$ comparison.

TOR reduced but did not eliminate the EC bias between the two temperature protocols. As a fraction of total carbon, the TOR EC measured with the He4-550 protocol is $1.23 \pm 0.11$ times higher than the TOR EC measured with the He4-700 protocol (the bias in the TOT EC was $1.48 \pm 0.17$ for the ten samples analyzed by both TOR and TOT). Similar to TOT, a larger bias is observed in the TOR EC of the wood smoke samples $(1.30 \pm 0.09)$ than for the non-wood-smoke samples $(1.13 \pm 0.03)$. These results are in contrast to Chow et al. (2004) who found no bias in TOR EC measured with different temperature protocols. One significant difference between the two studies is the EC loading of the filters; the peak EC loading of our filters was about $8 \mu \mathrm{g}$ $\mathrm{C} / \mathrm{cm}^{2}$, significantly lower than those considered by Chow et al. (2004).
The TOR EC is roughly a factor of two higher than TOT EC for both temperature protocols. This means that the split defined by TOR occurs earlier in the analysis cycle than the TOT split. Chow et al. (2004) also report substantially higher TOR EC than TOT EC. An earlier optical split increases the likelihood that non-light-absorbing carbon evolves after the OC/EC split. However, the ratio of TOR to TOT EC was similar for both temperature protocols (1.9 for He4-550 and 2.2 for He4-700). Therefore, factors other than just slip of non-light-absorbing carbon could contribute to the differences between TOR- and TOT-EC.

\section{DISCUSSION AND CONCLUSIONS}

Different types of carbon evolve during thermal-optical analysis of an ambient aerosol sample. Since the OC/EC split is optically defined, it is important to understand the optical properties of these different types of carbon. In this paper we have classified this carbon into three categories: non-light-absorbing carbon, light-absorbing pyrolyzed carbon (PC), and light-absorbing, native elemental carbon (EC). Our results show that changing the temperature protocol affects the relative rate at which these different types of carbon evolve during thermal-optical analysis. This, in turn, alters the optically defined OC/EC split and the measured EC ("EC"). These ideas provide a framework for understanding why different temperature protocols measure different amounts of EC.

The evolution of different types of carbon must be carefully considered when trying to select an optimum temperature protocol. First, the results presented here suggest $\mathrm{PC}$ and EC co-evolve from the He/Ox mode for any temperature protocol. Therefore, it is incorrect to view the post-split carbon as native EC. For ambient samples analyzed using a protocol with a high peak Hemode temperature (He4-870), a mixture of PC and EC evolves after the split. For ambient samples analyzed with a low peak He-mode temperature (He4-550), a mixture of PC, EC, and nonlight-absorbing carbon can evolve after the split. The optimum protocol will be one in which the amount of post-split carbon is equivalent to the mass of native EC on the filter. This will only occur when the optical properties of the post-split carbon are the same as EC. However, our results show that the optical properties of EC and PC can be significantly different.

For samples analyzed with the He4-870 protocol, a larger fraction of the EC evolves earlier in the analysis cycle-in the He4 temperature step-compared to samples analyzed with the He4-700 protocol. In Pittsburgh, $k_{\mathrm{PC}}$ is greater than $k_{\mathrm{EC}}$, so shifting EC evolution to earlier in the analysis cycle (relative to PC) reduces the measured EC. This explains why the He4870 protocol measures $20-30 \%$ less post-split carbon ("EC") than the He4-700 protocol. Similarly, too low a peak He-mode temperature-as in the He4-550 protocol—can result in nonlight-absorbing carbon evolving in the $\mathrm{He} / \mathrm{Ox}$ mode after the OC/EC split, biasing the EC high relative to the protocols with higher peak He-mode temperatures, (e.g., the He4-700 or 
He4-870 protocols). This effect likely contributes significantly to the He4-550 protocol measuring about 50\% more "EC" than the He4-700 protocol on wood smoke dominated ambient samples. These results are with the transmission-defined split. Similar differences are also seen with a reflectance-based OC/EC split, driven by the same changes in evolution of the different types of carbon (relative to each other) brought about by a changing temperature protocol.

Selection of the temperature protocol does influence relative biases created by either premature evolution of EC (accentuated by higher peak He-mode temperatures) or slip of non-lightabsorbing carbon into the He/Ox mode (possible at lower peak He-mode temperatures). When selecting a temperature protocol, one seeks to balance the two competing effects. The sample composition, both organic and inorganic, can also influence evolution of carbon from filters (Novakov and Corrigan 1995; Yu, $\mathrm{Xu}$, and Yang 2002). Since both premature EC evolution and slip of non-light-absorbing carbon in the He/Ox mode likely occur over a range of temperatures in addition to potentially significant temporal and spatial changes in sample composition, it is unlikely that a single He-mode peak temperature can be specified at which both problems disappear.

Of the three protocols considered here, the He4-700 protocol appears to yield the best estimate of EC for the Pittsburgh samples. Although PC and EC co-evolve in the He/Ox mode of samples analyzed with He4-700 protocol (as they do in all of the protocols considered here), the average attenuation coefficient of the post-split He/Ox carbon for samples analyzed with the He4-700 protocol $\left(28 \mathrm{~m}^{2} / \mathrm{g}-\mathrm{C}\right)$ is only slightly higher than the expected value of $k_{\mathrm{EC}}$ obtained from our washed samples $\left(22.9 \mathrm{~m}^{2} / \mathrm{g}-\mathrm{C}\right)$. Our experiments with levoglucosan and SOA suggest that positive bias in measured EC (due to slip of nonlight-absorbing carbon into the $\mathrm{He} / \mathrm{Ox}$ mode) may not be a significant problem for the He4-700 protocol.

For other locations and sample compositions, one could select a temperature protocol that minimizes loss of EC in the He-mode and slip of non-light-absorbing carbon into the He/Ox mode using repeated analysis and solvent extraction. However, this may be impractical for routine monitoring applications such as the STN. A way out could be to track the laser signal throughout the analysis, which can indicate premature loss of EC. Comparing the attenuation coefficient of the post-split carbon using a reference value for $k_{\mathrm{EC}}$ (or even an upper limit of $k_{\mathrm{EC}}$ ) can also help estimate any bias. For research projects that analyze a small number of samples, a combination of solvent-extraction with thermal/optical analysis is highly recommended.

\section{REFERENCES}

Birch, M. E., and Cary, R. A. (1996). Elemental Carbon-Based Method for Monitoring Occupational Exposures to Particulate Diesel Exhaust, Aerosol Sci. Technol. 25(3):221-241.

Bond, T. C., and Bergstrom, R. W. (2006). Light Absorption by Carbonaceous Particles: An Investigative Review, Aerosol Sci. Technol 40:1-41.
Cary, R. A. (2003). Personal Communication.

Chow, J. C., Watson, J. G., Chen, L. W. A., Arnott, W. P., and Moosmuller, H. (2004). Equivalence of Elemental Carbon by Thermal/Optical Reflectance and Transmittance with Different Temperature Protocols, Environ. Sci. Technol 38(16):4414-4422.

Chow, J. C., Watson, J. G., Crow, D., Lowenthal, D. H., and Merrifield, T. (2001). Comparison of IMPROVE and NIOSH Carbon Measurements, Aerosol Sci. Technol. 34(1):23-34.

Chow, J. C., Watson, J. G., Pritchett, L. C., Pierson, W. R., Frazier C. A., and Purcell, R. G. (1993). The DRI Thermal Optical Reflectance Carbon Analysis System-Description, Evaluation and Applications in United-States Air-Quality Studies, Atmos. Environ Part A-General Topics 27(8):11851201.

Conny, J. M., Klinedinst, D. B., Wight, S. A., and Paulsen, J. L. (2003). Optimizing Thermal-Optical Methods for Measuring Atmospheric Elemental (black) Carbon: A Response Surface Study, Aerosol Sci. Technol. 37(9):703723.

Fuller, K. A., Malm, W. C., and Kreidenweis, S. M. (1999). Effects of Mixing on Extinction by Carbonaceous Particles, J. Geophys. Res.-Atmospheres 104(D13):15941-15954.

Fung, K. (1990). Particulate Carbon Speciation by $\mathrm{MnO}_{2}$ Oxidation, Aerosol Sci. Technol 12(1):122-127.

Fung, K., Chow, J. C., and Watson, J. G. (2002). Evaluation of OC/EC Speciation by Thermal Manganese Dioxide Oxidation and the IMPROVE Method, J. Air \& Waste Manag. Assoc. 52(11):1333-1341.

Grieshop, A. P., Lipsky, E. M., Pekney, N. J., Takahama, S., and Robinson, A. L. (2005). Diesel and Gasoline Vehicle Gaseous and Fine Particle Emission Factors Measured in a Highway Tunnel: Effects of Fleet Composition and Season, Atmos. Environ. in press.

Gundel, L. A., Dod, R. L., Rosen, H., and Novakov, T. (1984). The Relationship Between Optical Attenuation and Black Carbon Concentration for Ambient and Source Particles, Sci. Total Environ 36(JUN):197-202.

Huntzicker, J. J., Johnson, R. L., Shah, J. J., and Cary, R. A. (1982). Analysis of Organic and Elemental Carbon in Ambient Aerosols by a Thermal-Optical Method. In G. T. Wolff and R. L. Klimisch. Particulate Carbon: Atmospheric Life Cycle, New York, Plenum, 79-88.

Kerminen, V. M., Makela, T. E., Ojanen, C. H., Hillamo, R. E., Vilhunen, J. K., Rantanen, L., Havers, N., VonBohlen, A., and Klockow, D. (1997). Characterization of the Particulate Phase in the Exhaust from a Diesel Car, Environ. Sci. Technol. 31(7):1883-1889.

Liousse, C., Cachier H., and Jennings, S. G. (1993). Optical and Thermal Measurements of Black Carbon Aerosol Content in Different EnvironmentsVariation of the Specific Attenuation Cross-Section, Sigma (Sigma), Atmos. Environ. Part A-General Topics 27(8):1203-1211.

Malissa, H., Puxbaum, H., and Pell, E. (1976). Simultaneous RelativeConductometric Determination of Carbon and Sulfur in Dusts. Fresenius Zeitschrift Fur Analytische Chemie (Fresenius' Journal of Analytical Chemistry, and now Analytical and Bioanalytical Chemistry) 282(2):109-113.

Malm, W. C., Sisler, J. F., Huffman, D., Eldred, R. A., and Cahill, T. A. (1994). Spatial and Seasonal Trends in Particle Concentration and Optical Extinction in the United-States, J. Geophys. Res._Atmospheres 99(D1):13471370 .

NIOSH (1999). Elemental Carbon (Diesel Particulate): Method 5040. (http://www.cdc.gov/niosh/nmam/pdfs/5040f3.pdf).

Novakov, T., and Corrigan, C. E. (1995). Thermal Characterization of Biomass Smoke Particles, Mikrochim Acta 119(1-2):157-166.

Peterson, M. R., and Richards, M. H. (2002). Thermal-Optical-Transmittance Analysis for Organic, Elemental, Carbonate, Total Carbon, and OCX2 in PM2.5 by the EPA/NIOSH Method. Symposium on Air Quality Measurement Methods and Technology-2002, San Francisco, Air \& Waste Management Association.

Petzold, A., Kopp, C., and Niessner, R. (1997). The Dependence of the Specific Attenuation Cross-Section on Black Carbon Mass Fraction and Particle Size, Atmos. Environ. 31(5):661-672. 
Schauer, J. J., Kleeman, M. J., Cass, G. R., and Simoneit, B. R. T. (2001). Measurement of Emissions from Air Pollution Sources. 3. C-1-C-29 Organic Compounds from Fireplace Combustion of Wood, Environ. Sci. Technol. 35(9):1716-1728.

Schauer, J. J., Mader, B. T., Deminter, J. T., Heidemann, G. Bae, M. S., Seinfeld, J. H., Flagan, R. C., Cary, R. A., Smith, D., Huebert, B. J., Bertram, T., Howell, S., Kline, J. T., Quinn, P., Bates, T., Turpin, B., Lim, H. J., Yu, J. Z., Yang, H., and Keywood, M. D. (2003). ACE-Asia Intercomparison of a Thermal-Optical Method for the Determination of Particle-Phase Organic and Elemental Carbon, Environ. Sci. Technol. 37(5):993-1001.

Schmid, H., Laskus, L., Abraham, H. J., Baltensperger, U., Lavanchy, V., Bizjak, M., Burba, P., Cachier, H., Crow, D., Chow, J., Gnauk, T., Even, A., ten Brink, H. M., Giesen, K. P., Hitzenberger, R., Hueglin, E., Maenhaut, W., Pio, C., Carvalho, A., Putaud, J. P., Toom-Sauntry, D., and Puxbaum, H. (2001). Results of the "Carbon Conference" International Aerosol Carbon Round Robin Test Stage I, Atmos. Environ. 35(12):21112121.

Simoneit, B. R. T., Schauer, J. J., Nolte, C. G., Oros, D. R., Elias, V. O., Fraser, M. P., Rogge, W. F., and Cass, G. R. (1999). Levoglucosan, a Tracer for Cellulose in Biomass Burning and Atmospheric Particles, Atmos. Environ. 33(2):173-182.
Subramanian, R., Khlystov, A. Y., Cabada, J. C., and Robinson, A. L. (2004). Positive and Negative Artifacts in Particulate Organic Carbon Measurements with Denuded and Undenuded Sampler Configurations, Aerosol Sci. Technol. 38(S1):27-48.

Turpin, B. J. (1989). Secondary Formation of Organic Aerosol: Investigation of the Diurnal Variations of Organic and Elemental Carbon (PhD Thesis). Environmental Science and Engineering, Oregon Graduate Center. Beaverton, Oregon, USA.

Weingartner, E., Saathoff, H., Schnaiter, M., Streit, N., Bitnar, B., and Baltensperger, U. (2003). Absorption of Light by Soot Particles: Determination of the Absorption Coefficient by Means of Aethalometers, J. Aerosol Sci. 34(10): 1445-1463.

Wittig, A. E., Anderson, N., Khlystov, A. Y., Pandis, S. N., Davidson, C. I., and Robinson, A. L. (2004). Pittsburgh Air Quality Study Overview and Initial Scientific Findings, Atmos. Environ. 38(20):3107-3125.

Yang, H., and Yu, J. Z. (2002). Uncertainties in Charring Correction in the Analysis of Elemental and Organic Carbon in Atmospheric Particles by Thermal/Optical Methods, Environ. Sci. Technol. 36(23):5199-5204.

Yu, J. Z., Xu, J. H., and Yang, H. (2002). Charring Characteristics of Atmospheric Organic Particulate Matter in Thermal Analysis, Environ. Sci. Technol. 36(4):754-761. 